\title{
Shock formation in the dispersionless Kadomtsev-Petviashvili equation
}

\author{
T. Grava ${ }^{1,2}$, C. Klein ${ }^{3}$, and J. Eggers ${ }^{1}$ \\ ${ }^{1}$ School of Mathematics, University of Bristol, University Walk, Bristol BS8 1TW, \\ United Kingdom \\ ${ }^{2}$ SISSA, Via Bonomea 265, I-34136 Trieste, Italy \\ ${ }^{3}$ Institut de Mathématiques de Bourgogne, Université de Bourgogne, 9 avenue Alain \\ Savary, 21078 Dijon Cedex, France
}

\begin{abstract}
The dispersionless Kadomtsev-Petviashvili (dKP) equation $\left(u_{t}+\right.$ $\left.u u_{x}\right)_{x}=u_{y y}$ is one of the simplest nonlinear wave equations describing twodimensional shocks. To solve the dKP equation we use a coordinate transformation inspired by the method of characteristics for the one-dimensional Hopf equation $u_{t}+u u_{x}=0$. We show numerically that the solutions to the transformed equation develops singularities at later times with respect to the solution of the dKP equation. This permits us to extend the dKP solution as the graph of a multivalued function beyond the critical time when the gradients blow up. This overturned solution is multivalued in a lip shape region in the $(x, y)$ plane, where the solution of the dKP equation exists in a weak sense only, and a shock front develops. A local expansion reveals the universal scaling structure of the shock, which after a suitable change of coordinates corresponds to a generic cusp catastrophe. We provide a heuristic derivation of the shock front position near the critical point for the solution of the dKP equation, and study the solution of the dKP equation when a small amount of dissipation is added. Using multiple-scale analysis, we show that in the limit of small dissipation and near the critical point of the dKP solution, the solution of the dissipative dKP equation converges to a Pearcey integral. We test and illustrate our results by detailed comparisons with numerical simulations of both the regularized equation, the dKP equation, and the asymptotic description given in terms of the Pearcey integral.
\end{abstract}




\section{Introduction}

Perhaps the best known example of a singularity in an evolution equation is the formation of jump discontinuities of the density and of the velocity field in the Euler equations of compressible gas dynamics. As these discontinuities propagate, they are known as shock waves. In the case of a planar shock front, the problem can be reduced to a one-dimensional equation for the velocity alone [30] (a so-called simple wave). The resulting wave profile overturns to form an s-shaped curve, the point where the gradient first becomes infinite (known as the gradient catastrophe) corresponds to the formation of a shock. From the overturned solution the physical solution can be reconstructed by inserting a jump discontinuity (the shock). The shock solution is a weak solution of the equation, which satisfies additional conditions motivated by physical considerations 31]. This shock solution is also found by taking the limit of vanishing viscosity in the dissipative form of the equations, yielding a weak solution (see [6] for conservation laws in one space dimension and [29, 19] for hyperbolic equations in several space dimensions).

The existence of such gradient catastrophe points has been proved in [2, 33] for hyperbolic equations in many space dimensions. However, to the best of our knowledge, if the initial condition depends on two or three spatial variables, little is known about the two or three-dimensional spatial structure of the shock near the blow-up points of the gradients. In particular, it would be interesting to know the self-similar structure of the solution both before and after shock formation [14]. A rare instance of where we have a more or less complete understanding of a higher dimensional singularity is the spatial structure of caustics of wave fronts in the approximation of geometrical optics [4, 41]. Two-dimensional wave breaking has also been studied in [43], using a simple kinematic equation, for which an exact implicit solution is available.

In this paper, we study the formation of two-dimensional shocks in a simple nonlinear wave equation known variously as the dispersionless Kadomtsev-Petviashvili (dKP) equation [22], or the Zabolotskaya-Khokhlov (ZK) equation [49]. The equation has the advantage that its one-dimensional form, the Hopf equation, has only one family of characteristics. The dKP equation can be seen as a long wavelength version of the original Kadomtsev-Petviashvili (KP) equation [22]:

$$
\left(u_{t}+u u_{x}+u_{x x x}\right)_{x}= \pm u_{y y}
$$

but with the highest order dispersive term $u_{x x x}$ dropped, namely

$$
\left(u_{t}+u u_{x}\right)_{x}= \pm u_{y y}
$$

The subscript denotes the derivative with respect to the variable. With a + sign on the right hand side, 1.1 is known as the KPI equation, or as the KPII equation 
in the opposite case. However, in the case of the dKP equation the two signs are equivalent under the transformation $u \rightarrow-u$ and $x \rightarrow-x$, and for the remainder of this manuscript we will consider only the positive sign. Depending on context, the KP equation describes wave profiles for layers of inviscid fluid of finite depth, waves in plasmas, or the propagation of sound beams in nonlinear media.

While the Cauchy problem for the KP equation is globally well-posed in a suitable space [40, by dropping the dispersive term, the dKP equation becomes a nonlocal scalar conservation law in two space variables. Even for smooth initial data, the solution remains smooth only for finite time. In [46] it is shown that the solution of the dKP equation is locally well posed in the Sobolev space $H^{s}, s>2$, so that for $s \geq 4$ one has classical solutions. Particular solutions of the dKP equations have been obtained with several techniques [16, 17, 27, 13, 45]. The Cauchy problem for the dKP equation and shock formation have been studied recently in [34, 35, 38, 37], using the inverse scattering transformation, which relies on the integrability of the dKP inherited from the KP equation [47, 50].

To sketch a derivation of the dKP equation, we follow the original derivation of the KP equation [22]. We start from the Hopf equation

$$
u_{t}+u u_{x}=0
$$

for a wave field $u$, with only a convective non-linearity. This is the simplest model equation describing wave steepening and shock formation. In a frame of reference moving at the sound speed $c$, a simple wave can be shown to be described by (1.2) [30]. Assuming a weak $y$-dependence, we add a small correction $\psi$ on the right hand side of $(1.2)$

$$
u_{t}+u u_{x}=\psi \text {. }
$$

For a wave of small amplitude, the second term in the above equation can be neglected. Assuming a dispersion relation $\omega=k c=\sqrt{k_{x}^{2}+k_{y}^{2}} c$, one obtains in a frame of reference moving along the $x$-axis with velocity $c$ that $\omega=k c-k_{x} c \approx c k_{y}^{2} /\left(2 k_{x}\right)$. For (1.3) to match this dispersion relation, we must have $\psi_{x} \approx c u_{y y} / 2$. Taking the $x$-derivative on both sides of $(1.3)$ we obtain

$$
\left(u_{t}+u u_{x}\right)_{x}=-\frac{c}{2} u_{y y}
$$

Rescaling $x \rightarrow-x u \rightarrow-u$ and $y \rightarrow \sqrt{2 / c} y$, one arrives at the equation

$$
\left(u_{t}+u u_{x}\right)_{x}=u_{y y}
$$

Note that in spite of its name, the dKP equation (1.4) contains dispersion, and only the highest order dispersive term has been dropped relative to (1.1). Other contexts in which (1.4) is used are described in [8]. 
The Hopf equation $(1.2)$ is solved by observing that the velocity is constant along characteristic curves $x(\xi, t)$, given by [9]:

$$
x(\xi, t)=u_{0}(\xi) t+\xi .
$$

Thus for any initial condition $u(x, 0)=u_{0}(x)$, one finds an exact solution $u(x, t)=u_{0}(\xi)$ in implicit form. Wave breaking occurs when two characteristics cross, which always occurs when the initial condition has negative slope. A shock first forms along the characteristic originating from the point $\xi_{c}$ of greatest negative slope by absolute value, where the solution $u(x, t)$ has a point of blow up of the gradient.

Thus if one expands the initial condition about $\xi_{c}$, one finds that the profile assumes a characteristic s-shape [14]:

$$
\Delta x-\Delta u \Delta t+t_{c}^{4} u^{\prime \prime \prime}\left(\xi_{c}\right) \Delta u^{3} / 6=0,
$$

where $\Delta u=u-u_{c}$, and $\Delta x=x-x_{c}-u_{c}\left(t-t_{c}\right)$. For $\Delta t=t-t_{c}>0$ (after shock formation), the profile has become multivalued. Balancing the three terms in (1.6), one sees directly that $\Delta u$ must be of order $\Delta t^{1 / 2}$, and so $\Delta x$ of oder $\Delta t^{3 / 2}$ [43, 14].

If one solves (1.4) with an initial condition which depends on $y$, the equation can no longer be solved with the method of characteristics. The idea underlying this paper is that the dependence on the $y$-coordinate is weak, so the structure of the solution is essentially the same as before, but the different stages of overturning are "unfolded" in the $y$-direction [39]. This means that effectively the singularity time becomes a function of $y$. If we choose the origin such that a singularity occurs at $y=0$ first, and expand $t_{c}$ in a Taylor series near $y=0$, we obtain $t_{c}(y)=t_{c}(0)+a y^{2}+O\left(y^{3}\right)$, with $a>0$ a constant and $t_{c}(0) \equiv t_{c}$. This means that $\Delta t=t-t_{c}-a y^{2} \equiv \bar{t}-a y^{2}$, and the two-dimensional wave breaking is governed by the scalings

$$
\Delta u \sim \bar{t}^{1 / 2}, \quad \Delta x \sim \bar{t}^{3 / 2}, \quad \Delta y \sim \bar{t}^{1 / 2} .
$$

In this paper we will show that the scalings 1.7 indeed describe the similarity structure of wave breaking in the dKP equation.

The estimates (1.7) imply that $\Delta y \gg \Delta x$ near the shock, consistent with our assumption of a slow variation in the $y$-direction. The central idea of our paper is to use this insight to generalize the characteristic transformation (1.5) to allow for a slow $y$-dependence:

$$
\left\{\begin{array}{l}
u(x, y, t)=F(\xi, y, t) \\
x=t F(\xi, y, t)+\xi
\end{array}\right.
$$

Applying transformation (1.8) to (1.4) results in a $\mathrm{PDE}$ for $F(\xi, y, t)$ which we will study in the next section (see equation (2.8)) ; the initial condition for $F$ is given by

$$
F(x, y, 0)=u_{0}(x, y)
$$


Note that if the initial data $u_{0}(x, y)$ has no $y$-dependence, (1.8) yields the exact characteristic solution with $F(x, y, t)=u_{0}(x)$ as described before; in particular, $F$ is $y$ and time-independent. As in the method of characteristics, the solution $u(x, y, t)$ of the dKP equation encounters a gradient catastrophe when the transformation $x=$ $t F(\xi, y, t)+\xi$ defining $\xi=\xi(x, y, t)$ is not invertible, namely when $t F_{\xi}(\xi, y, t)+1=0$. Our numerical results show that as a result of the unfolding $(1.8)$, the function $F(\xi, y, t)$ remains regular at the time $t_{c}$ of shock formation of the solution $u(x, y, t)$ of the dKP equation. Moreover, our numerics indicate the derivatives of $F$ remain bounded for times substantially beyond $t_{c}$. However, since $F$ satisfies a nonlinear equation (see (2.8) below), we believe that $F$ will typically develop a singularity for some time $t>t_{c}$; we give an example of such a singularity in a particular case.

Manakov and Santini [35, 38] have proposed a transformation for analysing the gradient catastrophe of $\mathrm{dKP}$ equation which is superficially similar to ours, which is motivated by the inverse scattering transform. Their transformation differs from ours by a factor of 2 in front of the unfolding term:

$$
\left\{\begin{array}{l}
u(x, y, t)=\tilde{F}(\zeta, y, t) \\
x=2 t \tilde{F}(\zeta, y, t)+\zeta \\
\tilde{F}(\zeta, y, 0)=u_{0}(\zeta, y)
\end{array}\right.
$$

as a result, the transformation does not unfold the overturned profile if there is no $y$-dependence. In fact, transformation of the Hopf equation leads to the same equation $\tilde{F}_{t}-\tilde{F} \tilde{F}_{\zeta}=0$ as before, but with propagation in the opposite direction, and with the same initial data $\tilde{F}(\zeta, 0)=u_{0}(\zeta)$. This means that for $y$-independent initial data localized in the $x$-direction, $\tilde{F}(\zeta, t)$ will experience a gradient catastrophe before $u(x, t)$ does, if the initial profile is steeper on the left than on the right. The same remains true for solutions of the full dKP equation with localized initial data: we checked numerically that for the initial data considered in this manuscript, i.e. the $x$-derivative of a Schwartz function, the function $\tilde{F}(\zeta, y, t)$ suffers a gradient catastrophe before a gradient catastrophe occurs in the original profile $u(x, y, t)$.

To further illustrate the difference between the two parameterizations, note that combining 1.8 and 1.10 one finds $F$ in terms of $\tilde{F}$ :

$$
\left\{\begin{array}{l}
F(\xi, y, t)=\tilde{F}(\zeta, y, t) \\
\xi=t \tilde{F}(\zeta, y, t)+\zeta
\end{array}\right.
$$

or $\tilde{F}$ in terms of $F$ :

$$
\left\{\begin{array}{l}
\tilde{F}(\zeta, y, t)=F(\xi, y, t) \\
\zeta=-t F(\xi, y, t)+\xi
\end{array}\right.
$$

If we assume that $\tilde{F}(\zeta, y, t)$ has no singularities and that $2 t \tilde{F}_{\zeta}(\zeta, y, t)+1>0$ in some time interval $\left[0, t^{\prime}\right]$, then it follows from 1.10 that the solution $u(x, y, t)$ of the dKP 
equation is regular in the same time interval. But since we also have $t \tilde{F}_{\zeta}(\zeta, y, t)+1>0$, it follows from (1.11) that $F(\xi, y, t)$ is regular in $\left[0, t^{\prime}\right]$ as well.

On the other hand, assuming that $F(\xi, y, t)$ is regular and $t F_{\xi}(\xi, y, t)+1>0$ in some time interval $\left[0, t^{\prime}\right]$, it follows from 1.8 that once again $u(x, y, t)$ is regular in $\left[0, t^{\prime}\right]$. However, this does not imply that $\tilde{F}(\zeta, y, t)$ is regular, since it may happen that $-t F_{\xi}(\xi, y, t)+1=0$ for some $t \in\left(0, t^{\prime}\right]$, even though $t F_{\xi}(\xi, y, t)+1>0$ for all $t \in\left[0, t^{\prime}\right]$. This argument shows that $\tilde{F}(\zeta, y, t)$, as defined by 1.10 , might encounter singularities even before $u(x, y, t)$ does.

Our formulation allows us to find spectrally accurate solutions to $F=F(\xi, y, t)$, from which $u(x, y, t)$ can easily be reconstructed. The alternative would be to use numerical methods for hyperbolic equations which remain stable even after the formation of shocks [32]. However, these methods introduce numerical dissipation near the shock, which renders the solution inaccurate. These sources of inaccuracy can be avoided using our transformation. The main results of this paper are the following:

- in section 2 we describe the solution of the dKP equation by using a transformation inspired by the method of characteristics and by [35]. This transformation reduces the Cauchy problem for the dKP equation to the Cauchy problem for the function $F(\xi, y, t)$ introduced in (1.8), which is regular beyond $t_{c}$.

- in section 3 we study the singularity formation in the solution to the dKP equation as done in [35], 38]. We then show that the local structure of the dKP solution near the point of gradient catastrophe, in a suitable system of coordinates, is equivalent to the unfolding of an $A_{2}$ singularity. We derive the self-similar structure of the lip-shaped domain where the solution of the dKP equation becomes multivalued.

- In section 4 we give a heuristic derivation of the shock front position near the critical point of the solution of the dKP equation, and study the solution of the dKP equation when dissipation is added (called the dissipative dKP equation). Using multiple-scale analysis, we show that in the limit of small dissipation and near the critical point of the dKP solution, the solution of the dissipative dKP equation converges to a Pearcey integral.

- In section 5 we compare our analysis with detailed numerical simulations. Solutions for initial data with and without symmetry with respect to $y \mapsto-y$ are studied. It is shown that our numerical approach allows to continue dKP solutions to a second gradient catastrophe, well after the first catastrophe has occurred. We find no indication for blow-up of the solution to the transformed dKP equation. 


\section{Solution by characteristic transformation}

We consider the Cauchy problem for the dKPI equation

$$
\begin{cases}\left(u_{t}+u u_{x}\right)_{x} & =u_{y y} \\ u(x, y, t=0) & =u_{0}(x, y), \quad x, y \in \mathbb{R}, t \in \mathbb{R}^{+} .\end{cases}
$$

Since we are interested mainly in local properties of the solution, we will assume that $u_{0}(x, y)$ is in the Schwartz class, namely it is smooth and decreases rapidly at infinity. Equation (2.1) can also be written in the evolutionary form

$$
u_{t}+u u_{x}=\partial_{x}^{-1} u_{y y}
$$

where $\partial_{x}^{-1} f(x) \equiv \int_{-\infty}^{x} f\left(x^{\prime}\right) d x^{\prime}$. This has the form of a nonlocal conservation law

$$
u_{t}+\nabla \mathbf{f}=0, \quad \mathbf{f}=\frac{u^{2}}{2} \mathbf{e}_{x}-\partial_{x}^{-1} u_{y} \mathbf{e}_{y}
$$

with $\mathbf{e}_{x}$ and $\mathbf{e}_{y}$ unit vectors in the $x$ and $y$ directions. As a result,

$$
\int_{\mathbb{R}^{2}} u(x, y, t) d x d y=\int_{\mathbb{R}^{2}} u_{0}(x, y) d x d y
$$

Similarly,

$$
\left(u^{2}\right)_{t}+\left[2 u^{3} / 3-\left(\partial_{x}^{-1} u_{y}\right)^{2}\right]_{x}+\left(2 u \partial_{x}^{-1} u_{y}\right)_{y}=0
$$

and hence the $L^{2}$ norm is also a conserved quantity:

$$
M(t) \equiv \int_{\mathbb{R}^{2}} u^{2}(x, y, t) d x d y=\int_{\mathbb{R}^{2}} u_{0}^{2}(x, y) d x d y .
$$

Since the left hand side of 2.1 is a total derivative, solutions have to satisfy the constraint

$$
\int_{\mathbb{R}} u_{y y}(x, y, t) d x=0, \quad t>0 .
$$

If the initial data does not satisfy such constraint, a low decays at infinity occurs for $t>0$ even for initial data in the Schwartz class. This is a manifestation of the infinite speed of propagation in the dKP equation. For this reason we choose initial data such that

$$
\int_{\mathbb{R}} u_{0}(x, y) d x=0
$$

for all $y$, so that the dynamical constraint is satisfied also at $t=0$. After these preliminaries we transform the dKP equation using (1.8), to find an equation for $F(\xi, y, t)$. 
Proposition 2.1 The equations (1.8) give a solution to the dKP equation with smooth initial data $u_{0}(x, y)$ in implicit form, if the function $F(\xi, y, t)$ satisfies the equation

$$
\left(\frac{F_{t}+t F_{y}^{2}}{1+t F_{\xi}}\right)_{\xi}=F_{y y}
$$

with initial data

$$
F(x, y, 0)=u_{0}(x, y)
$$

Proof Differentiating the second equation in 1.8 with respect to $x, t$ and $y$ we find

$$
\xi_{x}=\frac{1}{\Delta}, \quad \xi_{t}=-\frac{F+t F_{t}}{\Delta}, \quad \xi_{y}=\frac{t F_{y}}{\Delta}
$$

where we have defined $\Delta=1+t F_{\xi}$. Thus the derivatives of $u$ with respect to the variables are

$$
u_{t}=F_{\xi} \xi_{t}+F_{t}=\frac{F_{t}-F F_{\xi}}{\Delta}
$$

and

$$
u_{x}=\frac{F_{\xi}}{\Delta}, \quad u_{y}=\frac{F_{y}}{\Delta}
$$

Now the Hopf equation becomes

$$
0=u_{t}+u u_{x}=\frac{F_{t}}{\Delta}
$$

which confirms that $F$ is time-independent in this case. Differentiating 2.12 a second time, we find

$$
u_{y y}=\left(\frac{F_{y}}{\Delta}\right)_{y}-\left(\frac{F_{y}}{\Delta}\right)_{\xi} \frac{t F_{y}}{\Delta}=\frac{1}{\Delta}\left[F_{y y}-\left(\frac{t F_{y}^{2}}{\Delta}\right)_{\xi}\right]
$$

after some manipulations. But this means that if $u(x, y, t)$ satisfies the dKP equation (2.1), $F(\xi, y, t)$ satisfies (2.8) with initial condition (2.9).

We rewrite the equation (2.8) in the evolutionary form

$$
F_{t}=\partial_{\xi}^{-1} F_{y y}+t\left(\partial_{\xi}^{-1} F_{y y} F_{\xi}-F_{y}^{2}\right)
$$

where $\partial_{\xi}^{-1}$ is the inverse of a derivation. We observe from the above equation that the nonlinear terms are multiplied by the time $t$ and this show that for small times the nonlinear effects are damped. This observation qualitatively explains the fact that the function $F(\xi, y, t)$ develops a singularity after $u(x, y, t)$ becomes singular.

For the remainder of this paper we will focus on solutions to the transformed equation 2.8. We observe that 2.8 also conserves the integrals over $F$ and $F^{2}$, which we will use to check our numerics. Namely for $n$ integer one has

$$
\int_{\mathbb{R}^{2}} u^{n} d x d y=\int_{\mathbb{R}^{2}} F^{n} \Delta d \xi d y=\int_{\mathbb{R}^{2}} F^{n} d \xi d y+\frac{t}{n+1} \int_{\mathbb{R}^{2}}\left(F^{n+1}\right)_{\xi} d \xi d y=\int_{\mathbb{R}^{2}} F^{n} d \xi d y .
$$




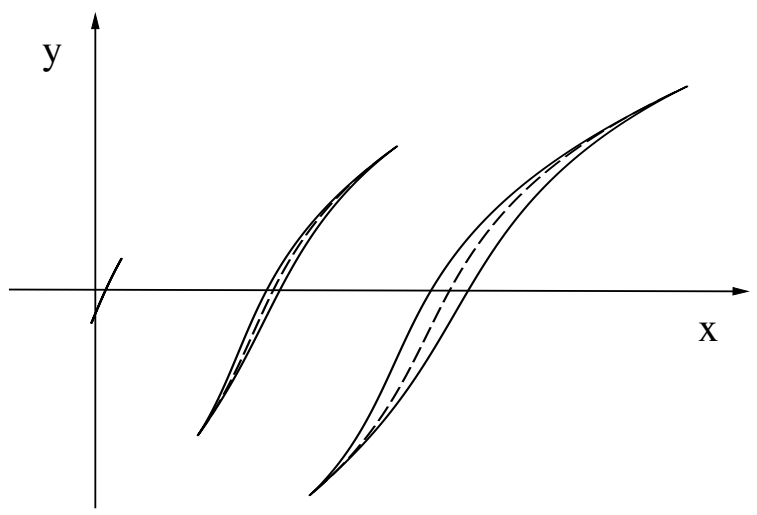

Figure 1. A typical sequence of wave breaking, as described by (3.1), showing the lip-shaped domain inside which the wave overturns. The singularity first appears at the origin, then spreads rapidly in the direction 3.3 . The scale of the lip is $\vec{t}^{3 / 2}$ in the $x$-direction, and $\vec{t}^{1 / 2}$ in the $y$-direction. Full lines are solutions of $\Delta=0$ at $\bar{t}=0.01,0.1$, and 0.4 , while the dashed line is the shock front, which has to be inserted in accordance with the shock condition (4.5), to be discussed in Section 4 below.

In particular, conservation of the $L^{2}$ norm $(2.6)$ gives the constraint

$$
\int_{\mathbb{R}^{2}} F^{2}(\xi, y, t) d \xi d y=\int_{\mathbb{R}^{2}} u_{0}^{2}(x, y, 0) d x d y
$$

The transformation (1.8) has been constructed so as to unfold the overturned profile onto the initial condition in the case of a $y$-independent initial condition. It is thus intuitive that if the overturning is modulated in the $y$-direction, it is unfolded onto a function $F(\xi, y, t)$ which shows no overturning, and having a weak dependence on $y$ and $t$ only.

\section{Overturning of the profile}

For generic initial data the solution of the dKP equation encounters a gradient catastrophe at points where the transformation (1.8) is not invertible [35]

$$
\Delta(\xi, y, t) \equiv 1+t F_{\xi}(\xi, y, t)=0
$$

and consequently the gradients $u_{x}$ and $u_{y}$ go to infinity, cf. 2.12). This is illustrated in Fig. 1 for generic initial data, based on the local description to be developed below. The singular time $t_{c}$ where the gradient catastrophe occurs first is the smallest $t$ such that (3.1) holds. Since for $t<t_{c}$ the quantity $\Delta(\xi, y, t)$ has a definite sign in the $\xi$ and $y$ plane, $\Delta\left(\xi, y, t_{c}\right)$ must be a zero as well as an extremum: $\Delta=\Delta_{\xi}=\Delta_{y}=0$. Thus 
the two-dimensional gradient catastrophe is characterized by the equations:

$$
\begin{aligned}
& 1+t F_{\xi}(\xi, y, t)=0 \\
& F_{\xi \xi}=0 \\
& F_{\xi y}=0 \\
& u(x, y, t)=F(\xi, y, t) \\
& x=t F(\xi, y, t)+\xi .
\end{aligned}
$$

The first three equations of 3.2 determine the coordinates $\xi_{c}, y_{c}$, and $t_{c}$ of the singularity in transformed variables, taken as the origin in Fig. 1. The $x$ and $u$ coordinates are recovered by substitution into the last two equations. One finds that $\left(t F_{y} \partial_{x}+\partial_{y}\right) u(x, y, t)=F_{y}<\infty$, hence there is no gradient catastrophe in the transversal direction characterized by the vector field

$$
t F_{y} \mathbf{e}_{x}+\mathbf{e}_{y}
$$

see Fig. 1. For generic initial conditions, the second derivatives of $\Delta$ will be nonzero at the gradient catastrophe:

$$
F_{\xi \xi \xi}\left(\xi_{c}, y_{c}, t_{c}\right) \neq 0, \quad F_{\xi \xi y}\left(\xi_{c}, y_{c}, t_{c}\right) \neq 0 \quad F_{\xi y y}\left(\xi_{c}, y_{c}, t_{c}\right) \neq 0
$$

The conditions $(3.2), 3.4)$ correspond to a cusp singularity in the notation of [1], and will be found to describe the generic singularity for the dKP solution. The condition that $F$ remains smooth, and thus the right hand side $F_{y y}$ of 2.8 is finite, results in the additional constraints

$$
F_{t}^{c}+t_{c}\left(F_{y}^{c}\right)^{2}=0, \quad F_{\xi t}^{c}=0, \quad F_{t y}^{c}+2 t_{c} F_{y y}^{c} F_{y}^{c}=0
$$

where with a super-script we indicate the derivatives evaluated at the critical point.

We now give a local description of the two-dimensional wave front $u(x, y, t)$, based on expanding $F(\xi, y, t)$ near the gradient catastrophe described by 3.2 . Our numerical simulations confirm that $F(\xi, y, t)$ remains smooth in the $(\xi, y)$ plane not only near the first singularity, but well beyond. The region where the wave is multivalued has the typical lip shape also seen in the caustic surface of light waves near the cusp catastrophe [41]. We will show them to be self-similar with width $\bar{t}^{3 / 2}$ in the horizontal direction and $t^{1 / 2}$ in the transversal direction where $\bar{t}=t-t_{c}$, as done in [35]. The same scalings have been observed in [43] in the context of the 2-dimensional kinematic wave equation.

In order to illustrate the way in which (1.8) unfolds the singularity, it is instructive to consider a family of exact solutions to 2.1 obtained in [36]:

$$
u(x, y, t)=\frac{1}{\sqrt{t}} B\left(x-\frac{y^{2}}{4 t}-2 u t\right),
$$


where $B$ is an arbitrary function of one variable. The validity of $(3.6)$ can be checked explicitly by substitution. Clearly $(3.6)$ can be re-parameterized in the form

$$
u(x, y, t)=\frac{1}{\sqrt{t}} B\left(\zeta-\frac{y^{2}}{4 t}\right), \quad x=2 \sqrt{t} B\left(\zeta-\frac{y^{2}}{4 t}\right)+\zeta,
$$

which shows that $\tilde{F}$, as defined by 1.10 , is

$$
\tilde{F}(\zeta, y, t)=\frac{1}{\sqrt{t}} B\left(\zeta-\frac{y^{2}}{4 t}\right)
$$

A singularity in the dKP solution occurs when the second equation in (3.7) is no longer invertible; the first time this occurs is the critical time $t_{c}>0$, determined by

$$
\sqrt{t_{c}}=\min _{\zeta \in \mathbb{R}}\left(-\frac{1}{2 B_{\zeta}}\right) \text {. }
$$

In order to write (3.6) in terms of our function $F(\xi, y, t)$, we use the double reparameterisation:

$$
\begin{aligned}
& u(x, y, t)=F(\xi, y, t), \quad x=t F(\xi, y, t)+\xi \\
& F(\xi, y, t)=\frac{1}{\sqrt{t}} B\left(\zeta-\frac{y^{2}}{4 t}\right), \quad \xi=\sqrt{t} B\left(\zeta-\frac{y^{2}}{4 t}\right)+\zeta .
\end{aligned}
$$

We observe that $F(\xi, y, t)$ has a singularity when the second transformation in (3.9) is no longer invertible, namely at a critical time for the transformed equation (2.8)

$$
t_{c}^{(F)}=4 t_{c}
$$

It is also straightforward to check that at $t_{c}, F(\xi, y, t)$ satisfies the constraints (3.5). Indeed one calculates directly from (3.9) that

$$
F_{t}=-\frac{B}{2 t^{\frac{3}{2}}} \frac{2 \sqrt{t} B^{\prime}+1}{\sqrt{t} B^{\prime}+1}+\frac{y^{2} B^{\prime}}{4 t^{\frac{5}{2}}} \frac{1}{\sqrt{t} B^{\prime}+1}, \quad F_{y}=-\frac{y B^{\prime}}{2 t^{\frac{3}{2}}\left(\sqrt{t} B^{\prime}+1\right)}
$$

so at the critical time one obtains the relations

$$
F_{t}^{c}=-\frac{y_{c}^{2}}{4 t_{c}^{3}}, \quad F_{y}^{c}=\frac{y_{c}}{2 t_{c}^{2}},
$$

which satisfy the first of the constraints in (3.5); the remaining constraints (3.5) are checked analogously. 


\subsection{Local analysis}

In order to study the solution near the gradient catastrophe we expand the generalized characteristic equation (1.8) in a Taylor series near $t_{c}, x_{c}, y_{c}, u_{c}$ and $\xi_{c}$. Part of the analysis below is already contained in [35], [38]. Introducing variables relative to the singularity as

$$
\bar{x}:=x-x_{c} \quad \bar{t}:=t-t_{c}, \quad \bar{y}:=y-y_{c}, \quad \bar{\xi}: \xi-\xi_{c},
$$

we have argued that $\bar{x} \sim \bar{t}^{3 / 2}$ and $\bar{y} \sim \bar{t}^{1 / 2}$. Since $\Delta u \sim \bar{t}^{1 / 2}$, it follows from the first equation of 1.8 that $\bar{\xi} \sim \bar{t}^{1 / 2}$. Thus to be consistent, we include all terms up to $O\left(\bar{t}^{3 / 2}\right)$ :

$$
\begin{aligned}
\bar{x} & =\bar{t}\left(F^{c}+t_{c} F_{t}^{c}\right)+\bar{t} \bar{y}\left(F_{y}^{c}+t_{c} F_{y t}^{c}\right)+t_{c}\left(F_{y}^{c} \bar{y}+\frac{1}{2} F_{y y}^{c} \bar{y}^{2}+\frac{1}{6} F_{y y y}^{c} \bar{y}^{3}\right) \\
& +\frac{t_{c}}{6} F_{\xi \xi \xi}^{c} \bar{\xi}^{3}+\frac{t_{c}}{2} F_{\xi \xi y}^{c} \bar{y} \bar{\xi}^{2}+\left(\frac{t_{c}}{2} F_{\xi y y}^{c} \bar{y}^{2}+F_{\xi}^{c} \bar{t}\right) \bar{\xi}+o\left(\bar{t}^{2}, \bar{y}^{4}, \bar{\xi}^{4}, \bar{t}\left(\bar{y}^{2}+\bar{\xi}^{2}\right)\right) .
\end{aligned}
$$

This suggests introducing the shifted variables (using $t_{c}=-1 / F_{\xi}^{c}$ ):

$$
\begin{aligned}
\zeta & =F_{\xi}^{c}\left(\bar{\xi}+\frac{F_{\xi \xi y}^{c}}{F_{\xi \xi \xi}^{c}} \bar{y}\right) \\
X & =\frac{1}{k}\left[\bar{x}-\bar{t}\left(F^{c}+t_{c} F_{t}^{c}\right)-\bar{t} \bar{y}\left(F_{y}^{c}+t_{c} F_{y t}^{c}\right)-t_{c}\left(F_{y}^{c} \bar{y}+\frac{1}{2} F_{y y}^{c} \bar{y}^{2}+\frac{1}{6} F_{y y y}^{c} \bar{y}^{3}\right)\right. \\
- & \left.\frac{1}{3} t_{c} \frac{\left(F_{\xi \xi y}^{c}\right)^{3}}{\left(F_{\xi \xi \xi}^{c}\right)^{2}} \bar{y}^{3}+\frac{1}{2} t_{c} \frac{F_{\xi \xi y}^{c} F_{\xi y y}^{c}}{F_{\xi \xi \xi}^{c}} \bar{y}^{3}+F_{\xi}^{c} \frac{F_{\xi \xi y}^{c}}{F_{\xi \xi \xi}^{c}} \bar{y} \bar{t}\right] \\
T & =\frac{1}{k}\left[\bar{t}+\frac{t_{c}^{2}}{2} \bar{y}^{2}\left(\frac{\left(F_{\xi \xi y}^{c}\right)^{2}}{F_{\xi \xi \xi}^{c}}-F_{\xi y y}^{c}\right)\right], \\
k & =\frac{t_{c}^{4} F_{\xi \xi \xi}^{c}}{6}
\end{aligned}
$$

so that in the variable $\zeta, 3.10$ takes the form

$$
-\zeta^{3}+T \zeta=X+o\left(\bar{t}^{2}, \bar{y}^{4}, \bar{\xi}^{4}, \bar{t}\left(\bar{y}^{2}+\bar{\xi}^{2}\right)\right) .
$$

Using the estimates $\bar{\xi} \sim \bar{y} \sim \bar{t}^{1 / 2}$ and $\bar{x} \sim \bar{t}^{3 / 2}$ identified previously, the scaling

$$
\begin{aligned}
& X \rightarrow \lambda X \\
& \bar{t} \rightarrow \lambda^{\frac{2}{3}} \bar{t} \\
& \bar{y} \rightarrow \lambda^{\frac{1}{3}} \bar{y} \\
& \zeta \rightarrow \lambda^{\frac{1}{3}} \zeta,
\end{aligned}
$$




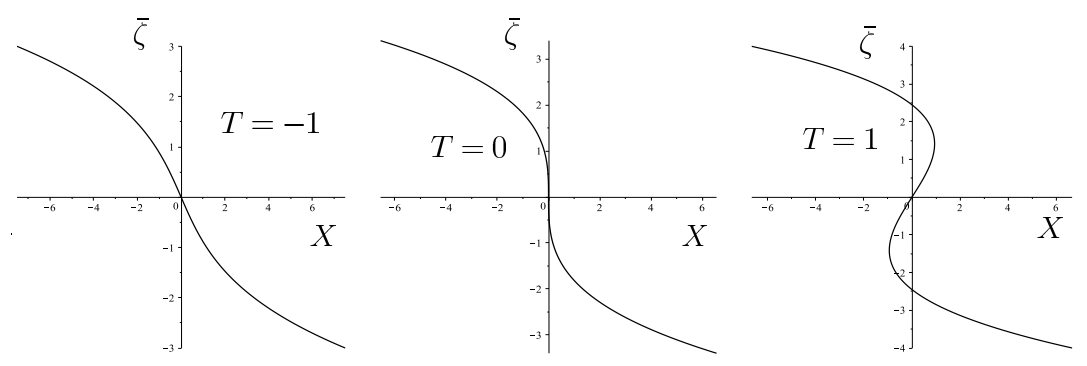

Figure 2. The universal s-curve described by (3.14); for $T>0$ the profile turns over to form a multivalued region.

in the limit $\lambda \rightarrow 0$ reduces $(3.12)$ to the universal s-curve

$$
-\zeta^{3}+T \zeta=X
$$

shown in Fig. 2. It is easy to confirm that the function $\zeta(X, T)$ defined by $(3.14)$ solves

$$
\zeta_{T}+\zeta \zeta_{X}=0
$$

with initial condition $\zeta(X, T=0)=(-X)^{\frac{1}{3}}$, completing our task of reducing 2.1) locally to the Hopf equation. A gradient catastrophe is encountered for $X=0, T=0$, and $\zeta=0$.

Using the identities (3.5), we can now calculate the solution to the dKP equation (2.1), valid near the singularity. To leading order in the limit $\lambda \rightarrow 0$, it is consistent to expand $u(x, y, t)$ to linear order in $\bar{\xi}, \bar{y}$ :

$$
u(x, y, t)-u_{c}=F(\xi, y, t)-F^{c} \simeq F_{\xi}^{c} \bar{\xi}+F_{y}^{c} \bar{y}=\zeta(X, T)+\bar{\beta} \bar{y}
$$

with

$$
\bar{\beta}=F_{y}^{c}-\frac{F_{\xi}^{c} F_{\xi \xi y}^{c}}{F_{\xi \xi \xi}^{c}} .
$$

Thus putting $\bar{u} \equiv u(x, y, t)-u_{c}$, from $(3.14)$ we find the local profile to be an s-curve, which has the universal similarity form:

$$
-(\bar{u}-\bar{\beta} \bar{y})^{3}+T(\bar{u}-\bar{\beta} \bar{y})=X .
$$

This is the central result of our theoretical analysis; the formula $(3.18)$ is the unfolding of an $A_{2}$ singularity. It is a complete description of the self-similar behavior of the dKP solution near its singularity for generic initial data. In the $y$-independent case, (3.18) coincides with the usual result (1.6). We now derive the form of this multivalued valued region in the $x, y$-plane, shown previously in Fig. 1 . 


\subsection{Multivalued region}

As seen in Fig. 2, the function $\zeta=\zeta(X, T)$, described by the cubic equation (3.14), becomes multivalued for $T>0$. From $\frac{\partial X}{\partial \zeta}=0$ it follows that

$$
T=3 \zeta^{2}, \quad \text { or } \quad \zeta= \pm \sqrt{T / 3}
$$

so that for $X$ in the interval

$$
-\frac{2}{3 \sqrt{3}} T^{3 / 2} \leq X \leq \frac{2}{3 \sqrt{3}} T^{3 / 2}
$$

the function $\zeta(X, T)$ is multivalued.

Reversing the coordinate transformations (3.11), we can write the first equation (3.19) in the form

$$
\bar{t}=\frac{1}{2} t_{c} \alpha\left(\bar{\xi}^{2}+2 \beta \bar{y} \bar{\xi}+\gamma \bar{y}^{2}\right)
$$

where we have introduced the constants

$$
\alpha=t_{c} F_{\xi \xi \xi}^{c}, \quad \beta=\frac{F_{\xi \xi y}^{c}}{F_{\xi \xi \xi}^{c}}, \quad \gamma=\frac{F_{\xi y y}^{c}}{F_{\xi \xi \xi}^{c}} .
$$

Alternatively, (3.20) could also have been derived from (3.1), and expanding $F$ in a power series around the singular point.

The $\bar{x}$-coordinate of the boundary of overturning can be found from $(3.10)$, which using 3.20 can be simplified to yield

$$
\begin{aligned}
& \bar{x}=-\alpha\left(\frac{1}{3} \bar{\xi}^{3}+\frac{\beta}{2} \bar{y} \bar{\xi}^{2}\right)+\bar{t}\left(F^{c}-t_{c}^{2}\left(F_{y}^{c}\right)^{2}\right)+ \\
& \bar{t} \bar{y}\left(F_{y}^{c}-2 t_{c}^{2} F_{y y}^{c} F_{y}^{c}\right)+t_{c}\left(F_{y}^{c} \bar{y}+\frac{1}{2} F_{y y}^{c} \bar{y}^{2}+\frac{1}{6} F_{y y y}^{c} \bar{y}^{3}\right) .
\end{aligned}
$$

Equations 3.20 and 3.22 describe a curve in the $(\bar{x}, \bar{y})$ plane, parameterized by $\bar{\xi}$. An example was shown previously in Fig. 1 for several values of $\bar{t}=t-t_{c}$, showing its characteristic "lip" shape [3].

The overturned region starts from the singular point and then expands, as seen in Fig. 1. To understand the scaling of this expansion, we introduce the independent variables

$$
\left\{\begin{aligned}
X_{1} & =\left[\bar{x}-\bar{t}\left(F^{c}-t_{c}^{2}\left(F_{y}^{c}\right)^{2}\right)-t_{c}\left(F_{y}^{c} \bar{y}+\frac{1}{2} F_{y y}^{c} \bar{y}^{2}\right)\right] \bar{t}^{-3 / 2} \\
Y_{1} & =\bar{y} \bar{t}^{-1 / 2}
\end{aligned}\right.
$$

Then the lip described by equations 3.20 and 3.22 is reduced to the timeindependent similarity form:

$$
\left\{\begin{array}{l}
\frac{1}{2} t_{c} \alpha\left(s^{2}+2 \beta Y_{1} s+\gamma Y_{1}^{2}\right)=1 \\
X_{1}=-\alpha\left(\frac{1}{3} s^{3}+\frac{1}{2} \beta s^{2} Y_{1}\right)+\delta_{1} Y_{1}+\frac{\delta_{2}}{6} Y_{1}^{3},
\end{array}\right.
$$




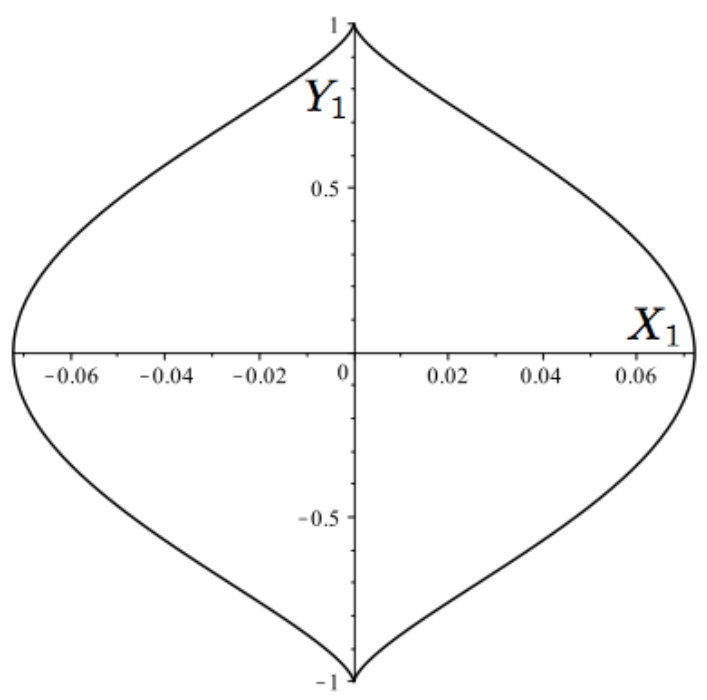

Figure 3. The symmetric lip according to 3.26 , ending in a cusp.

with the additional constants

$$
\delta_{1}=F_{y}^{c}-2 t_{c}^{2} F_{y y}^{c} F_{y}^{c}, \quad \delta_{2}=t_{c} F_{y y y}^{c} .
$$

This demonstrates that the gradient catastrophe in the dKP equation has a universal spatial signature, parameterized by the constants $\alpha, \beta, \gamma, \delta_{1}$, and $\delta_{2}$, all of which can be computed in terms of the initial data and its derivatives at the point of gradient catastrophe $\left(x_{c}, t_{c}, y_{c}\right)$. The scalings introduced in (3.23) imply that the lip expands as $\bar{t}^{3 / 2}$ in the propagation direction, and as $\bar{t}^{1 / 2}$ in the transversal direction, as announced previously. A characteristic feature is the cusp at the corner of the lip. This is seen most easily for initial data which is even in $y$, for which the description simplifies considerably. All odd derivatives in $y$ vanish, and we obtain

$$
\left\{\begin{array}{l}
\frac{1}{2} t_{c} \alpha\left(s^{2}+\gamma Y_{1}^{2}\right)=1 \\
X_{1}=-\frac{\alpha}{3} s^{3}
\end{array}\right.
$$

shown in Fig. 3. Analyzing the neighborhood of the point $s=0$, one finds directly that

$$
X_{1}= \pm \frac{\alpha}{3}\left(2 \bar{Y}_{1}\right)^{3 / 2}\left(\bar{Y}_{1}-Y_{1}\right)^{3 / 2}, \quad \bar{Y}_{1}=\sqrt{\frac{2}{t_{c} \alpha}},
$$

which is a generic $3 / 2$ cusp [15]. 


\section{Dissipative dKP equation and shock solutions}

The solution (3.18) constructed in the previous subsection is unphysical for $\bar{t}>0$, in that it does not assign a unique value of $u$ to every point $x, y$ in the plane. In principle, one can construct an infinity of single-valued solutions from it, by choosing different points at which to jump from one branch to the other. For conservation laws in many space dimensions, physically motivated constraints, known as generalized RankineHugoniot jump conditions, have been introduced. As result, a weak solution of the equations (usually called the inviscid shock), is singled out uniquely [33, 29].

Another way to select a unique solution after the singularity, is to consider a dissipative version of 2.1 with a viscous term added to it, which keeps the solution regular at all times. In the limit of vanishing viscosity $\epsilon$ these regular solutions are expected to converge to (3.18), with a particular jump condition being selected. In this case the shock is called the viscous shock. In the field of hyperbolic equations the problem of showing that the inviscid shock is equal to the viscous shock has generated a huge literature. We only mention some important references in one dimension [6, 18], and many space dimensions [19]. Below we give an heuristic derivation of the equivalence of the inviscid and viscous shock for the dKP equation.

We consider the dissipative form of the dKP equation

$$
\left.\left(u_{t}+u u_{x}-\epsilon\left(u_{x x}+c y_{y y}\right)\right)_{x}=u_{y y}, \quad u(x, y, t=0, \epsilon)\right)=u_{0}(x, y)
$$

with $c \geq 0$, which satisfies

$$
\frac{1}{2} \frac{\partial}{\partial t} \int_{\mathbb{R}^{2}} u^{2}(x, y, t) d x d y=-\epsilon\left(u_{x}^{2}+c u_{y}^{2}\right)<0 .
$$

For given $\epsilon$-independent initial data, the solution $u(x, y, t, \epsilon)$ of the dissipative equation (4.1) is expected to be approximated as $\epsilon \rightarrow 0$ and $t<t_{c}$ by the solution $u(x, y, t)$ of the dKP equation 2.1).

\subsection{Shock position}

On the other hand, (2.4) is still satisfied at finite $\epsilon$, so (smooth) solutions of (4.1) still conserve $u$ in the limit $\epsilon \rightarrow 0$. From the condition that $u$ be satisfied across a shock, we can use the generalized Rankine-Hugoniot jump conditions as in [33], which determines the shock position (see also [9]). Namely, if $v_{n}$ is the normal velocity of the shock, one obtains

$$
v_{n}\left(u_{1}-u_{2}\right)=(\mathbf{f} \cdot \mathbf{n})_{1}-(\mathbf{f} \cdot \mathbf{n})_{2},
$$

where $\mathbf{n}$ is the normal to the shock front, and indices 1 and 2 denote values in front and in the back of the shock, respectively. Assuming that the shock position is given 
by the curve $x_{s}(\bar{y}, \bar{t})$, and using the flux $\mathbf{f}$ from $(2.3)$, this yields

$$
\dot{x}_{s}\left(u_{1}-u_{2}\right)=\frac{u_{1}^{2}-u_{2}^{2}}{2}+\frac{\partial x_{s}}{\partial y} \int_{x_{2}}^{x_{1}} u_{y} d x
$$

where $x_{1 / 2}$ are $x$-values approaching the shock from the front and from behind, respectively.

Now the singular contribution to $u$ across the shock can be written in the form

$$
u=u_{2}+\left(u_{1}-u_{2}\right) \theta\left(\bar{x}-x_{s}(\bar{y}, \bar{t})\right)
$$

where $\theta(x)$ is the Heaviside function and $u_{1,2}$ become functions only of $y$ and $t$ on the shock front $\bar{x}=x_{s}(\bar{y}, \bar{t})$. Hence

$$
u_{y}=\left(u_{2}\right)_{y}+\left(u_{1}-u_{2}\right)_{y} \theta\left(\bar{x}-x_{s}(\bar{y}, \bar{t})\right)-\left(u_{1}-u_{2}\right) \frac{\partial x_{s}}{\partial y} \delta\left(\bar{x}-x_{s}(\bar{y}, \bar{t})\right),
$$

and from (4.4) the jump condition at the shock finally becomes

$$
\dot{x}_{s}=\frac{u_{1}+u_{2}}{2}-\left(\frac{\partial x_{s}}{\partial y}\right)^{2}
$$

Note that the shock speed in the $x$-direction is not only an average between $u$-values in front and in the back of the shock as for the Hopf equation, but on account of the right hand side of (2.1) an additional term arises.

Since we have mapped (2.1) locally to the Hopf equation 3.15, standard theory [9] tells us that the shock should be at $X=0$, according to 3.11 the equation for the front becomes

$$
\begin{aligned}
& x_{s}(\bar{y}, \bar{t})=\bar{t}\left(F^{c}+t_{c} F_{t}^{c}\right)+\bar{t} \bar{y}\left(F_{y}^{c}+t_{c} F_{y t}^{c}\right)+t_{c}\left(F_{y}^{c} \bar{y}+\frac{1}{2} F_{y y}^{c} \bar{y}^{2}+\frac{1}{6} F_{y y y}^{c} \bar{y}^{3}\right)+ \\
& \frac{1}{3} t_{c} \frac{\left(F_{\xi \xi y}^{c}\right)^{3}}{\left(F_{\xi \xi \xi}^{c}\right)^{2}} \bar{y}^{3}-\frac{1}{2} t_{c} \frac{F_{\xi \xi y}^{c} F_{\xi y y}^{c}}{F_{\xi \xi \xi}^{c}} \bar{y}^{3}-F_{\xi}^{c} \frac{F_{\xi \xi y}^{c}}{F_{\xi \xi \xi}^{c}} \bar{y} \bar{t}
\end{aligned}
$$

This equation indeed satisfies (4.5) to leading order, since

$$
\dot{x}_{s}(\bar{y}, \bar{t})=F^{c}+t_{c} F_{t}^{c}+\bar{y} t_{c} F_{y t}^{c}+\bar{\beta} \bar{y}
$$

and

$$
\left(\frac{\partial x_{s}}{\partial y}\right)^{2}=\left[t_{c}\left(F_{y}^{c}+F_{y y}^{c} \bar{y}\right)+O(\bar{t})\right]^{2}=-t_{c} F_{t}^{c}-t_{c} F_{t y}^{c} \bar{y}+O(\bar{t}),
$$

having used 3.5 . On the other hand, $\zeta_{ \pm}= \pm \sqrt{T}$ at $X=0$, and so according to 3.16

$$
\frac{u_{1}+u_{2}}{2}=F^{c}+\bar{\beta} \bar{y} .
$$

Combining the last three equations one can see that the approximate shock front 4.6) satisfies 4.5 to leading order. In Fig. 1 we have plotted 4.6 as the dashed line. 


\subsection{Shock structure}

Having found the shock position, we now investigate the inner structure of the shock, in case a small amount of viscosity is present. This is achieved by mapping (4.1) onto Burgers' equation [48, which in addition to 3.15 contains a dissipative contribution. We are looking for a solution $u(x, y, t ; \epsilon)$ of the dissipative dKP equation near the gradient catastrophe $\left(x_{c}, y_{c}, t_{c}\right)$ of the (inviscid) dKP equation. To this end we use the ansatz

$$
u(x, y, t ; \epsilon)=u_{c}+h(X, T ; \epsilon)+\bar{y} \bar{\beta},
$$

with $X$ and $T$ defined in 3.11. Using the same scalings as before, and balancing $u_{t} \propto \epsilon u_{x x}$, we are led to the multiscale expansion

$$
\begin{aligned}
& h(X, T ; \epsilon)=\lambda^{\frac{1}{3}} H(\mathcal{X}, \mathcal{T} ; \varepsilon)+O\left(\lambda^{\alpha}\right), \quad \alpha>\frac{1}{3}, \\
& X=\lambda \mathcal{X}, \quad T=\lambda^{\frac{2}{3}} \mathcal{T}, \quad \epsilon=\lambda^{\frac{4}{3}} \varepsilon, \quad \bar{y}=\lambda^{\frac{1}{3}} \mathcal{Y},
\end{aligned}
$$

and find the following theorem:

Theorem 4.1 Let $u(x, y, t ; \epsilon)=u_{c}+h(X, T ; \epsilon)+\bar{y} \bar{\beta}$ be a solution of the dissipative dKP equation (4.1) with $X$ and $T$ defined in (3.11). Suppose that for $\left|t-t_{c}\right|$ small the limit

$$
H(\mathcal{X}, \mathcal{T} ; \varepsilon)=\lim _{\lambda \rightarrow 0} \lambda^{-\frac{1}{3}} h\left(\lambda \mathcal{X}, \lambda^{2 / 3} \mathcal{T} ; \lambda^{\frac{4}{3}} \varepsilon\right)
$$

exists and the function $H(\mathcal{X}, \mathcal{T} ; \varepsilon)$ satisfies the asymptotic conditions

$$
H(\mathcal{X}, \mathcal{T} ; \varepsilon)=\mp|\mathcal{X}|^{\frac{1}{3}} \mp \frac{\mathcal{T}}{3}|\mathcal{X}|^{-\frac{1}{3}}+O\left(|\mathcal{X}|^{-\frac{5}{3}}\right), \quad|\mathcal{X}| \rightarrow \infty
$$

for each fixed $\mathcal{T} \in \mathbb{R}$. Then the function $H(\mathcal{X}, \mathcal{T} ; \varepsilon)$ satisfies the Burgers equation

$$
H_{\mathcal{T}}+H H_{\mathcal{X}}=\sigma H_{\mathcal{X X}}, \quad \sigma=\frac{\varepsilon}{k}\left(1+c\left(t_{c} F_{y}^{c}\right)^{2}\right)
$$

with $k$ defined in 3.11.

Proof Inserting 4.9 into the dissipative dKP equation one obtains

$$
\begin{aligned}
& \left(H_{\mathcal{T}}+H_{\mathcal{X}}-\frac{\varepsilon}{k}\left(1+\left(t_{c} F_{y}^{c}\right)^{2}\right) H_{\mathcal{X} \mathcal{X}}\right)_{\mathcal{X}}+\lambda^{-\frac{1}{3}} H_{\mathcal{X X}}\left(\frac{\partial X}{\partial t}-\left(\frac{\partial X}{\partial y}\right)^{2}+F_{c}+\bar{y}\left(F_{y}^{c}-F_{\xi}^{c} \frac{F_{\xi \xi y}^{c}}{F_{\xi \xi \xi}^{c}}\right)\right) \\
= & H_{\mathcal{T} \mathcal{X}} \frac{\partial T}{\partial y} \frac{\partial X}{\partial y}+\lambda^{\frac{1}{3}} H_{\mathcal{T} \mathcal{T}}\left(\frac{\partial T}{\partial y}\right)^{2}+\lambda k H_{\mathcal{T}} \frac{\partial^{2} T}{\partial y^{2}}+\lambda^{2 / 3} k H_{\mathcal{X}} \frac{\partial^{2} X}{\partial y^{2}}-\varepsilon\left(H_{\mathcal{X} \mathcal{X}}\left(\left(\frac{\partial X}{\partial y}\right)^{2}-\left(t_{c} F_{y}^{c}\right)^{2}\right)\right) \\
& -\frac{\varepsilon}{k}\left(\lambda^{1 / 3} H_{\mathcal{T} \mathcal{X}} \frac{\partial T}{\partial y} \frac{\partial X}{\partial y}+\lambda^{2 / 3} H_{\mathcal{T} \mathcal{T}}\left(\frac{\partial T}{\partial y}\right)^{2}+\lambda^{4 / 3} k H_{\mathcal{T}} \frac{\partial^{2} T}{\partial y^{2}}+\lambda k H_{\mathcal{X}} \frac{\partial^{2} X}{\partial y^{2}}\right)_{\mathcal{X}} .
\end{aligned}
$$


Using (3.11), the constraints (3.5), and the substitution 4.10) one arrives at the relation

$$
\left(H_{\mathcal{T}}+H H_{\mathcal{X}}-\frac{\varepsilon}{k}\left(1+c\left(t_{c} F_{y}^{c}\right)^{2}\right) H_{\mathcal{X} \mathcal{X}}\right)_{\mathcal{X}}=O\left(\lambda^{\frac{1}{3}}\right)
$$

which in the limit $\lambda \rightarrow 0$ shows that the derivative of 4.12 is equal to zero. In order to fix the integration constant we use the asymptotic condition 4.11.

We remark that the asymptotic condition (4.11) implies that the local solution near the point of singularity formation, matches the outer solution given by (3.14) and (3.16). We conclude that near the gradient catastrophe, up to the constant term $u_{c}$ as well as a term linear in $y$, in a suitable co-ordinate system the solution to the dissipative dKP equation reduces to the solution of the one-dimensional Burgers equation. We will argue below that the particular solution to the Burgers equations relevant near the critical point, and described by the asymptotic form 4.11, also satisfies the equation

$$
\mathcal{X}=H \mathcal{T}-H^{3}+6 \sigma H H_{\mathcal{X}}-4 \sigma^{2} H_{\mathcal{X}}
$$

4.2.1. Burgers equation. To find the local solution near the shock, let us recall the solution to Burgers' equation

$$
v_{t}+v v_{x}=\nu v_{x x}
$$

with initial data $v_{0}(x)$, where $\nu$ is a positive constant. An exact solution is obtained via the Cole-Hopf transformation [20],448] to give the formula:

$$
v(x, t, \nu)=-2 \nu \partial_{x} \log \int_{-\infty}^{\infty} e^{-\frac{G(\eta, x, t)}{2 \nu}} d \eta
$$

where

$$
G(\eta, x, t)=\int_{0}^{\eta} v_{0}(s) d s+\frac{(x-\eta)^{2}}{2 t} .
$$

For $\nu \rightarrow 0$ the leading contributions to the integral come from the neighborhood of the critical points of $G$, namely

$$
\left.\partial_{\eta} G(\eta, x, t)\right|_{\eta=\xi}=v_{0}(\xi)-\frac{x-\xi}{t}=0 .
$$

Let us assume first that there is only one such critical point, which means that using the method of the steepest descent [48], the integral can be approximated as

$$
\int_{-\infty}^{\infty} e^{-\frac{G(\eta, x, t)}{2 \nu}} d \eta \approx \sqrt{\frac{4 \pi \nu}{\partial_{\xi}^{2} G(\xi, x, t)}} e^{-\frac{G(\xi, x, t)}{2 \nu}},
$$

where $\xi=\xi(x, t)$ is a solution of 4.18). Direct evaluation of (4.16), using the characteristic condition (4.18), then yields the solution

$$
v(x, t, \nu)=v_{0}(\xi)+\nu \frac{v_{0}^{\prime \prime}(\xi) t}{\left(v_{0}^{\prime}(\xi) t+1\right)^{2}}+O\left(\nu^{2}\right),
$$


whose leading order contribution in the limit $\nu \rightarrow 0$ is the solution of the Hopf equation by characteristics. In addition, (4.19), contains a linear correction coming from the viscosity. Alternatively, the term linear in $\nu$ can also be obtained using perturbation theory.

The approximation (4.19) remains valid as long as the function $G(\eta, x, t)$ has an isolated generic critical point, before the appearance of a gradient catastrophe. However, after the critical triple point $\left(x_{c}, t_{c}\right)$ of the Hopf equation, where $v_{0}^{\prime}\left(\xi_{c}\right) t_{c}+1=$ 0 and $v_{0}^{\prime \prime}\left(\xi_{c}\right)=0,4.18$ has three solutions, as illustrated on the left of Fig. 5 below. Near this point $G(\eta, x, t)$ can be expanded in a Taylor series as

$$
\Delta G:=G(\eta, x, t)-G\left(\xi_{c}, c_{c}, t_{c}\right) \simeq v_{0}^{\prime \prime \prime}\left(\xi_{c}\right) \frac{\bar{\eta}^{4}}{4 !}-\bar{\eta}^{2} \frac{\bar{t}}{2 t_{c}^{2}}-\bar{\eta} \frac{\bar{x}-v_{c} \bar{t}}{t_{c}}+v_{c}\left(\bar{x}-v_{c} \bar{t}\right)+v_{c}^{2} \bar{t}
$$

where $\bar{x}=x-x_{c}, \bar{t}=t-t_{c}, \bar{\eta}=\eta-\xi_{c}, v_{c}=v_{0}\left(\xi_{c}\right)$ and $v_{0}^{\prime \prime \prime}\left(\xi_{c}\right)>0$. Thus near such critical point the solution of Burgers' equation can in the limit $\nu \rightarrow 0$ be approximated by

$$
v(x, t, \nu) \simeq v_{c}-2 \nu \partial_{x} \log \int_{-\infty}^{\infty} \exp \left[-\frac{1}{2 \nu}\left(v_{0}^{\prime \prime \prime}\left(\xi_{c}\right) \frac{\bar{\eta}^{4}}{4 !}-\bar{t} \frac{\bar{\eta}^{2}}{2 t_{c}^{2}}-\frac{\bar{\eta}}{t_{c}}\left(\bar{x}-v_{c} \bar{t}\right)\right)\right] d \bar{\eta} .
$$

Some rescaling leads to the following (see also [12])

Theorem 4.2 [21] Near a gradient catastrophe $\left(x_{c}, t_{c}\right)$ for the solution of the Hopf equation $v_{t}+v v_{x}=0$, the solution $v(x, t, \nu)$ of (4.15) admits the following expansion

$$
v(x, t, \nu)=v_{c}+\left(\frac{\nu}{\kappa}\right)^{1 / 4} U\left(\frac{\bar{x}-v_{c} \bar{t}}{\left(\kappa \nu^{3}\right)^{1 / 4}}, \frac{\bar{t}}{(\kappa \nu)^{1 / 2}}\right)+O\left(\nu^{1 / 2}\right)
$$

where $v_{c}=v\left(x_{c}, t_{c}\right), \kappa=t_{c}^{4} v_{0}^{\prime \prime \prime}\left(\xi_{c}\right) / 6$ and the function $U=U(a, b)$ is defined by

$$
U(a, b)=-2 \partial_{a} \log \int_{-\infty}^{+\infty} e^{-\frac{1}{8}\left(z^{4}-2 z^{2} b+4 z a\right)} d z
$$

Remark 4.3 The function $U(a, b)$ satisfies both the Burgers equation

$$
U_{b}+U U_{a}=U_{a a}
$$

and the non-linear ODE in the $a$-variable, containing $b$ as a parameter [5]

$$
a=U b-U^{3}+6 U U_{a}-4 U_{a a} .
$$

The behavior of $U(a, b)$ is illustrated in Fig. 4 for negative, positive, and vanishing values of reduced time $b$, performing the integral in 4.22 numerically. For large $|a|$ 


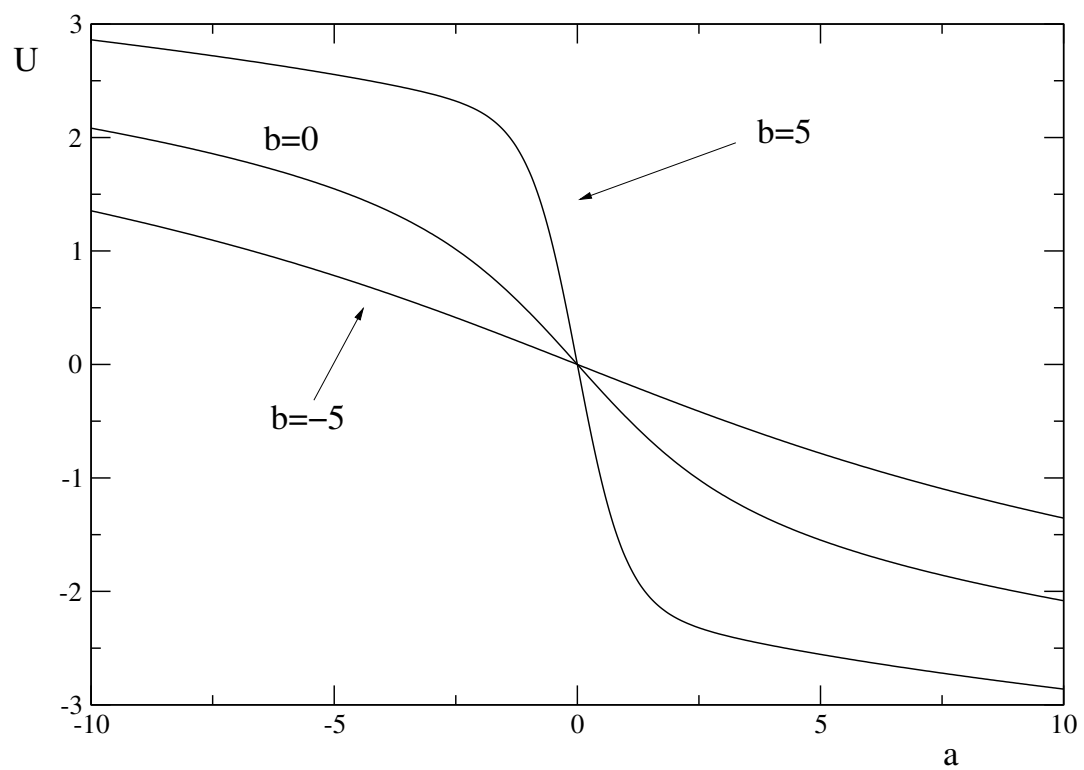

Figure 4. The Pearcey function $U(a, b)$, for three different values of $b$.

and fixed $b$ the integral 4.22 behaves as the root of the cubic equation (3.14) (see below)

$$
U(a, b)=\mp|a|^{\frac{1}{3}} \mp \frac{b}{3}|a|^{-\frac{1}{3}}+O\left(|a|^{-\frac{5}{3}}\right), \quad|a| \rightarrow \infty
$$

The integral in 4.22 is related to the standard Pearcey function [11, which describes the diffraction pattern near a cusp caustic [41], by a complex rotation. The relation 4.23 is convenient in deducing the asymptotic properties of $U(a, b)$; it follows from

$$
\int_{-\infty}^{\infty} \frac{d}{d z} e^{-\frac{1}{8}\left(z^{4}-2 z^{2} b+4 z a\right)} d z=0 .
$$

In catastrophe theory [44] the potential

$$
\Delta(z)=z^{4}-2 z^{2} b+4 z a
$$

(the weight in the exponent of (4.22) is the standard unfolding of the cusp catastrophe, which is a co-dimension 2 singularity. For $b<0$ (before the gradient catastrophe), there is only one critical point

$$
0=\frac{d \Delta}{d z}=4 z^{3}-4 z b+4 a
$$

which is the case we considered before (see Fig. 5). Evaluating $\Delta$ at the critical point 4.25 yields

$$
\Delta=-3 z^{4}+2 z^{2} b, \quad a=-z^{3}+z b .
$$



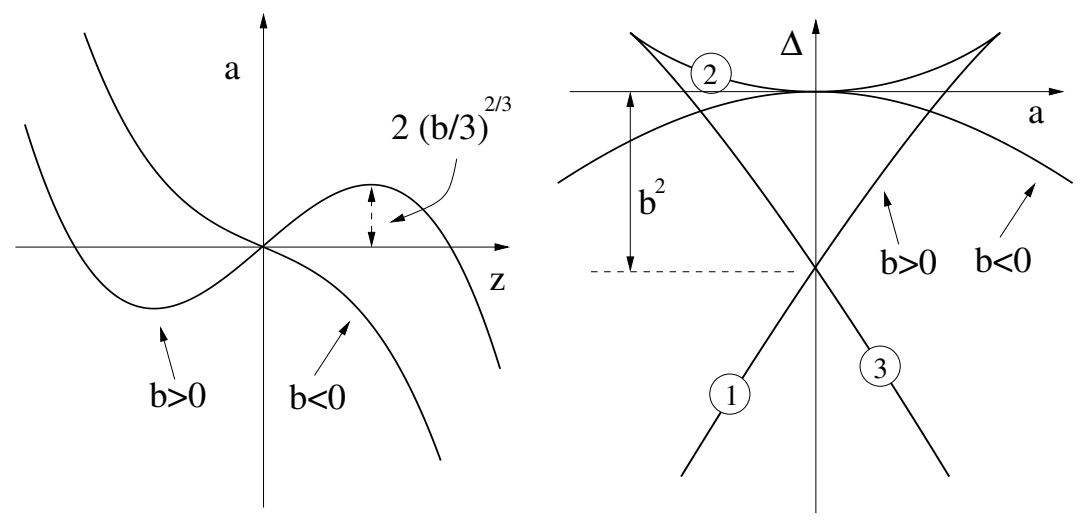

Figure 5. The critical contributions to the integral 4.22 near a cusp catastrophe, at constant reduced time $b$. On the left, the critical points; there is a unique solution for $b<0$, and three solutions for $|a| \leq 2(b / 3)^{3 / 2}$ if $b>0$. On the right, the argument $\Delta$ of the exponential; for $b<0$ there is a single contribution, for $b>0$ there are three contributions to a given value of $a$.

For $b<0$ this gives the single-valued curve shown on the right of Fig. 5, which leads to the solution (4.19).

If on the other hand $b>0$ (after the gradient catastrophe), in the range $|a| \leq 2(b / 3)^{3 / 2}$ there are three critical points. Thus the integral 4.22 has three contributions, with different values of $\Delta$ (cf. 4.26) , which lie on a swallowtail figure, as shown on the right of Fig. 5. The integral is dominated by the smallest value of $\Delta$, as long as the solutions are well separated. This means we must have $b \gg 1$ (cf. Fig. 5), or $\bar{t} / \epsilon^{1 / 2} \gg 1$. Closer to gradient catastrophe, a more sophisticated asymptotics is needed, or one has to evaluate the integral numerically, as we will do below. However, outside of the region $b \lesssim 1$, the integral is dominated by either solution $z_{1}$ or $z_{3}$. The changeover occurs for $a=0$, where $\Delta\left(z_{1}\right)=\Delta\left(z_{3}\right)$, namely on the line $\bar{x}-v_{c} \bar{t}=0$. This is exactly the shock front near the gradient catastrophe $\left(x_{c}, t_{c}\right)$.

4.2.2. Pearcey integral and dissipative dKP equation. Choosing $\lambda=\epsilon^{\frac{3}{4}}$ in Theorem 4.1 we obtain that the solution to the dissipative $\mathrm{dKP}$ equation satisfies in the rescaled variables 4.10 the Burgers equation 4.12 with $\varepsilon=1$. Furthermore for $t<t_{c}$ such solution is asymptotic to the Hopf solution (3.14). Combining these observations with Theorem 4.2 and remark 4.3 , we come up the following conjecture.

Conjecture 1 Let us consider the double scaling limit $\epsilon \rightarrow 0, x \rightarrow x_{c}, y \rightarrow y_{c}$ and $t \rightarrow t_{c}$ in such a way that the ratios

$$
\frac{X}{\epsilon^{3 / 4}}, \quad \frac{T}{\epsilon^{1 / 2}},
$$


remain bounded with $X$ and $T$ defined in (3.11). Then the solution $u(x, y, t ; \epsilon)$ of the dissipative dKP equation near the first singularity for the solution of the dKP equation is described by the expansion

$$
u(x, y, t ; \epsilon) \simeq u_{c}+\sigma^{1 / 4} U\left(\frac{X}{\sigma^{3 / 4}}, \frac{T}{\sigma^{1 / 2}}\right)+\bar{y} \bar{\beta}+O\left(\epsilon^{1 / 2}\right),
$$

where

$$
\sigma=\epsilon \frac{6\left(1+c\left(t_{c} F_{y}^{c}\right)^{2}\right)}{F_{\xi \xi \xi}^{c} t_{c}^{4}}
$$

and the function $U(a, b)$ is the Pearcey integral defined in (4.22).

For $y$-symmetric initial data the expression 4.27 reduces to the form

$$
u(x, y, t ; \epsilon) \simeq u_{c}+\sigma^{1 / 4} U\left(\frac{\bar{x}-u_{c} \bar{t}-t_{c} F_{y y}^{c} \bar{y}^{2} / 2}{k \sigma^{3 / 4}}, \frac{\bar{t}-t_{c}^{2} F_{\xi y y}^{c} \bar{y}^{2} / 2}{k \sigma^{1 / 2}}\right)+O\left(\epsilon^{1 / 2}\right),
$$

with $k$ defined in (3.11). The center of the (smooth) shock front is located at $X=0$, as found previously in the inviscid limit.

\section{Numerical solution}

In this section we present numerical solutions of the transformed version (2.8) of the dKP equation, which remain smooth well beyond the gradient catastrophe of the original equation (2.1), as we will demonstrate below. In addition, we treat the dissipative dKP equation (4.1), whose solutions are also observed to remain smooth. We use a Fourier method for the spatial dependence, and an exponential time differencing (ETD) scheme for the time dependence, as previously for the dKP equation [25].

Both equations are written in evolutionary form

$$
F_{t}=\partial_{\xi}^{-1} F_{y y}+t\left(F_{\xi} \partial_{\xi}^{-1} F_{y y}-F_{y}^{2}\right)
$$

and

$$
u_{t}+u u_{x}=\partial_{x}^{-1} u_{y y}+\epsilon\left(u_{x x}+c u_{y y}\right)
$$

with a small dissipation parameter $\epsilon$. In Fourier space, the antiderivatives $\partial_{\xi}^{-1}$ and $\partial_{x}^{-1}$ are represented as Fourier multipliers $-i / k_{\xi}$ and $-i / k_{x}$, respectively. Here $k_{\xi}, k_{x}, k_{y}$ are the dual Fourier variables of $\xi, x, y$ respectively, and the Fourier transform of a variable will be denoted by a hat. Thus (5.1) and $(5.2)$ can be written in the form

$$
\hat{u}_{t}=\mathcal{L} \hat{u}+\mathcal{N}(\hat{u})
$$


where $\mathcal{L}$ is a linear, diagonal operator, which is $i k_{y}^{2} / k_{\xi}$ for $(5.1)$, and $i k_{y}^{2} / k_{x}-\epsilon k_{x}^{2}$ for (5.2), and $\mathcal{N}(\hat{u})$ is a nonlinear term. The idea of the ETD scheme to be used here is to treat the linear part of $(5.3)$ exactly. We use the fourth order EDT method by Cox and Matthews [10], but other schemes offer a very similar performance [24].

To satisfy the constraint 2.7) on the initial condition, we choose initial data as the derivative of a function from the Schwarz space of rapidly decreasing smooth functions. This is well suited to a Fourier method, since a Schwarz function can be continued as a smooth periodic function to within our finite numerical precision. However, the nonlocality of (5.1) and (5.2) implies that solutions will develop tails with an algebraic decrease towards infinity. This follows already from the Green function of the linearized equations [26]. It was shown in [26, 24] that discontinuities at the boundaries of the computational domain can nevertheless be avoided by choosing a large enough domain, and one can achieve spectral accuracy (an exponential decrease of the numerical error with the number of Fourier modes) over the time scales considered.

The antiderivative in both (5.1) and (5.2) leads to Fourier multipliers which are singular in the limit of small wave numbers. These terms are regularized in Fourier space by adding a term of the order of the machine precision $\left(\sim 10^{-16}\right.$ here). In [26], the dKP equation (2.1) was solved for $\partial_{x}^{-1} u$, which is possible since solutions maintain the property of being the derivative of a Schwarz function. Together with an exponential integrator treating the term $i k_{y}^{2} / k_{x}$ explicitly, this addressed all numerical problems stemming from this singular operator.

However, an explicit treatment of all singular terms is not possible for (5.1), since $\mathcal{N}$ is singular as well, which leads to numerical problems for $k_{\xi} \rightarrow 0$. This can be addressed by applying a Krasny filter [28]: all Fourier coefficients with modulus smaller than some threshold (typically $10^{-10}$ ) will be put equal to 0 . In all cases considered, our numerical algorithm could now be continued well beyond the first gradient catastrophe. For longer times, the above mentioned algebraic tails will lead to a slower decrease of the Fourier coefficients and thus to numerical problems once the numerical errors are of the order of the Krasny filter. For long time computations, which are beyond the scope of the current paper, one would have to use considerably larger domains and higher resolutions, or alternatively a spectral approach as in [7].

The accuracy of the numerical solution to (2.8) was monitored via the decrease of the Fourier coefficients, and checking the conservation of the $L^{2}$ norm (cf. (2.6), 2.14)). To this end we compute

$$
\delta(t)=1-\frac{M(t)}{M(0)}
$$

whose time dependence will be a measure of the numerical error. As shown in [23, 25], the maximum error in $F$ may well be one to two orders of magnitude greater than $\delta$, but within these limits $\delta$ is nevertheless a reliable indicator of the accuracy, if the 


\begin{tabular}{|c|c|c|c|c|c|c|}
\hline Breaking event & Initial data & $t_{c}$ & $x_{c}$ & $y_{c}$ & $u_{c}$ & $\xi_{c}$ \\
\hline First & $-6 \partial_{x} \operatorname{sech}^{2} \sqrt{x^{2}+y^{2}}$ & 0.222 & 1.79 & 0 & 2.543 & 1.227 \\
\hline Second & $-6 \partial_{x} \operatorname{sech}^{2} \sqrt{x^{2}+y^{2}}$ & 0.300 & -2.033 & 0 & -2.48 & -1.289 \\
\hline
\end{tabular}

Table 1. Critical parameters for the first two wave breaking events, with symmetric initial data (5.5).

Fourier coefficients decrease sufficiently rapidly.

\subsection{Shock formation for symmetric initial data}

We begin with the simplest case of initial data symmetric with respect to $y \rightarrow-y$. We choose the same initial condition as [25],

$$
u_{0}(x, y)=-6 \partial_{x} \operatorname{sech}^{2} \sqrt{x^{2}+y^{2}}
$$

who solved the dKP equation (2.1) in its original form. Near the gradient catastrophe, (2.1) develops a discontinuity, and the numerical scheme employed in [25] breaks down. By contrast, using the transformed equation (2.8), we are able to reach the gradient catastrophe with much lower resolution (using serial instead of parallel computers), but are also able to continue the computation beyond the first and even secondary wavebreaking events. Beyond the gradient catastrophe, we identify the lines $\Delta=0$ along which the gradient of the solution blows up (cf. Fig. 1), and show that the solution of (2.8) yields the expected weak solution of dKP inside the lip region. We also show that the solution of 2.8 stays regular on time scales of order unity.

In [25], the first wave breaking event was observed at the critical time $t_{c}=$ $0.2216 \ldots$, see Table 1. Here we can identify $t_{c}$ directly from a solution of (2.8) by tracing the minimum of $\Delta$ over space. The first time this quantity vanishes or becomes just negative will be taken as the time $t_{c}$. We use $N_{x}=N_{y}=2^{9}$ Fourier modes for $x, y \in[-5 \pi, 5 \pi]^{2}$ and $N_{t}=1000$ time steps for $t \leq 0.23$. The first negative value of $\Delta$ is recorded for $t=0.222 \ldots$, which is in agreement with [25] to within the accuracy of at least two digits. However, the present calculation requires much lower resolution to reach similar accuracy $\left(N_{x}=N_{y}=2^{9}\right.$ compared to $N_{x}=N_{y}=2^{15}$ in [25]), and accuracy can easily be improved. For example, after determining the critical time to a certain accuracy, one uses the required resolution in time close to the previously determined $t_{c}$. This allows to determine the critical time with the same precision as the solution to (2.8), i.e., with the accuracy of the Krasny filter chosen here to be equal to $10^{-10}$. For our purposes an accuracy of the order of $10^{-3}$ will be sufficient.

The location of the critical point was identified in [25] as $x_{c}=1.79 \ldots$ and $y_{c}=0$. Here it is calculated for $t=t_{c}$ by first finding the minimum $\xi_{c}=1.227 \ldots, y_{c}=0$ of 

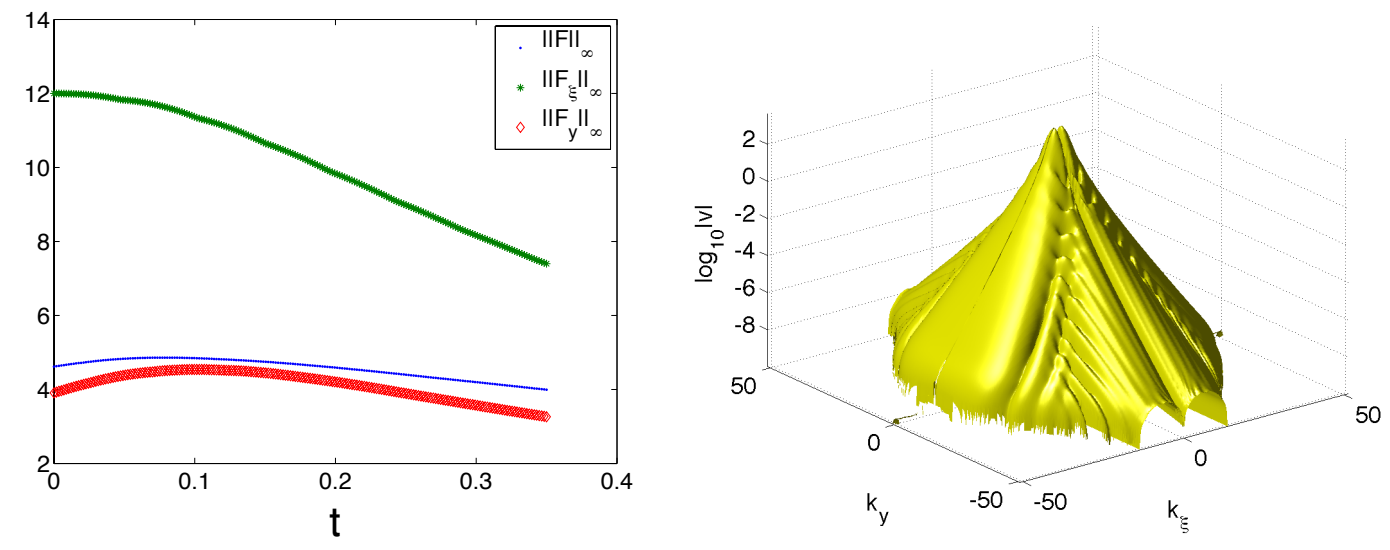

Figure 6. Measures of the smoothness of the solution to (2.8) with initial data (5.5). On the left, the time dependence of the maximum norm of $F$, as well as of $F_{\xi}$ and $F_{y}$; all decay for long times. On the right, the Fourier coefficients of the solution for $t=0.32$.

$\Delta$, where $F\left(\xi_{c}, y_{c}, t_{c}\right)=2.543 \ldots$ Then, using $(3.2)$, we find $x_{c}=\xi_{c}+t_{c} F\left(\xi_{c}, y_{c}, t_{c}\right)=$ $1.792 \ldots$, again in excellent agreement with our previous result [25], estimated to be correct to at least two digits.

However, the solution $F$ of $(2.8)$ stays perfectly regular well beyond the critical time $t_{c}$ of the dKP solution $u(x, y, t)$, as seen in Fig. 6. On the left, we show that the maximum norms of the first derivatives of $F$ remain bounded and smooth at $t_{c}$, and even decay for long times (of course, the derivatives of the original variable $u(x, y, t)$ diverge at a gradient catastrophe). On the right, for $t=0.32$ we demonstrate exponential decay of the Fourier coefficients to the level of the Krasny filter, as expected for a smooth function. The relative $L^{2}$ norm $\delta(t)$ (cf. (5.4)) is conserved to the order of $10^{-14}$. On account of the algebraic decay of the solution in Fourier space, the computation cannot be run for much longer than $t=0.35$ at the current resolution. To be able to do so using a Fourier method, larger domains and higher resolution would be needed. However, there is no indication that the solution of (2.8) itself develops a singularity.

Thus it is possible to continue the computation beyond the first wave breaking event, and to identify the second event, which occurs for negative $x$. This is of course not possible in the case of direct integration of (2.1) as in [25], where the numerical method fails at the first wave breaking. We use $N_{x}=2^{9}, N_{y}=2^{11}$ Fourier modes and $N_{t}=5000$ time steps for $t \leq 0.32$. Proceeding as for the first break-up in tracing the minimum of $\Delta(\xi, y, t)$, we find $\tilde{t}_{c}=0.300 \ldots$ and $\tilde{x}_{c}=-2.033 \ldots$, see Table 1 .

The corresponding profile $u(x, y, t)$ can be seen in Fig. 7 on the left. It is obtained by plotting $F(\xi, y, t)$ (shown on the right) as a function of $x=\xi+t F(\xi, y, t)$, as 

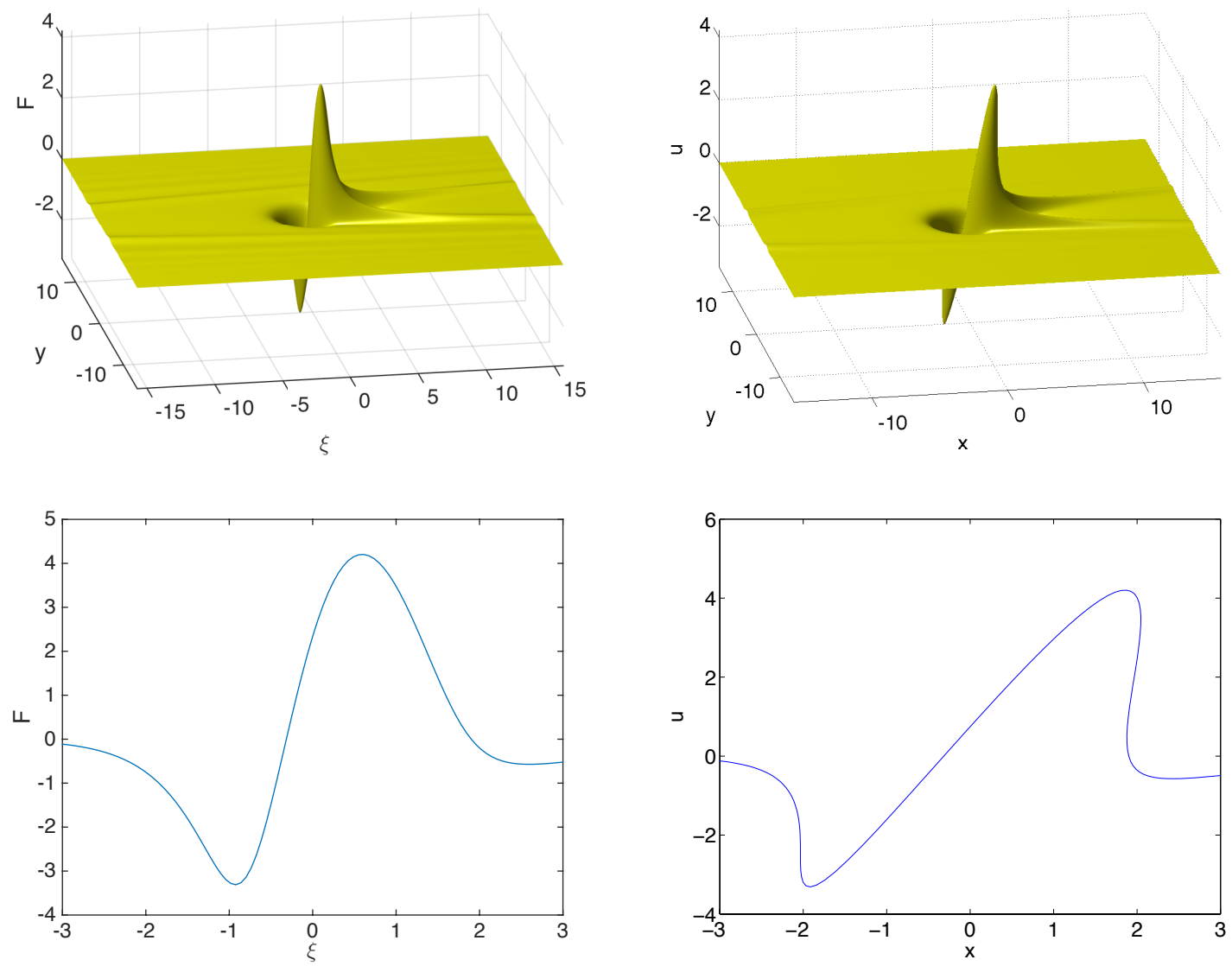

Figure 7. Profiles obtained from a solution of the transformed equation (2.8) at $t=0.300$, time of the second wave breaking event. On the left, the original solution $F(\xi, y, t)$ for initial data 5.5) on the right, the profile $u(x, y, t)$ obtained using the transformation (1.8). The slices along the plane $y=0$ (bottom) make it clear that the profile $u(x, y, t)$ has overturned near $x=2$ (first breaking), and is at the point of breaking near $x=-2$ (second breaking). The profile of $F(\xi, y, t)$ remains smooth and single valued.

required by (1.8). For $t>t_{c}$ in a neighborhood of the blow-up point, one has that $x=\xi+t F(\xi, y, t)$ is not invertible as a function of $\xi(x, y, t)$. However we can still perform a parametric plot of $u(x, y, t)$, which becomes a multivalued function in the region near the first critical point $\left(x_{c}, 0, t_{c}\right)$. This is even clearer from the cut along the $y=0$-axis shown on the bottom (recall that the critical points are all on the $x$ axis since the initial data are symmetric with respect to $y \rightarrow-y$, and since the dKP equation preserves this symmetry). Thus as for the solution to the Hopf equation via the characteristic method, a nonphysical solution which has overturned is obtained in the shock region. It is clear from the corresponding cut through $F(\xi, y, t)$ shown on the bottom left that $F$ remains smooth and single valued. 

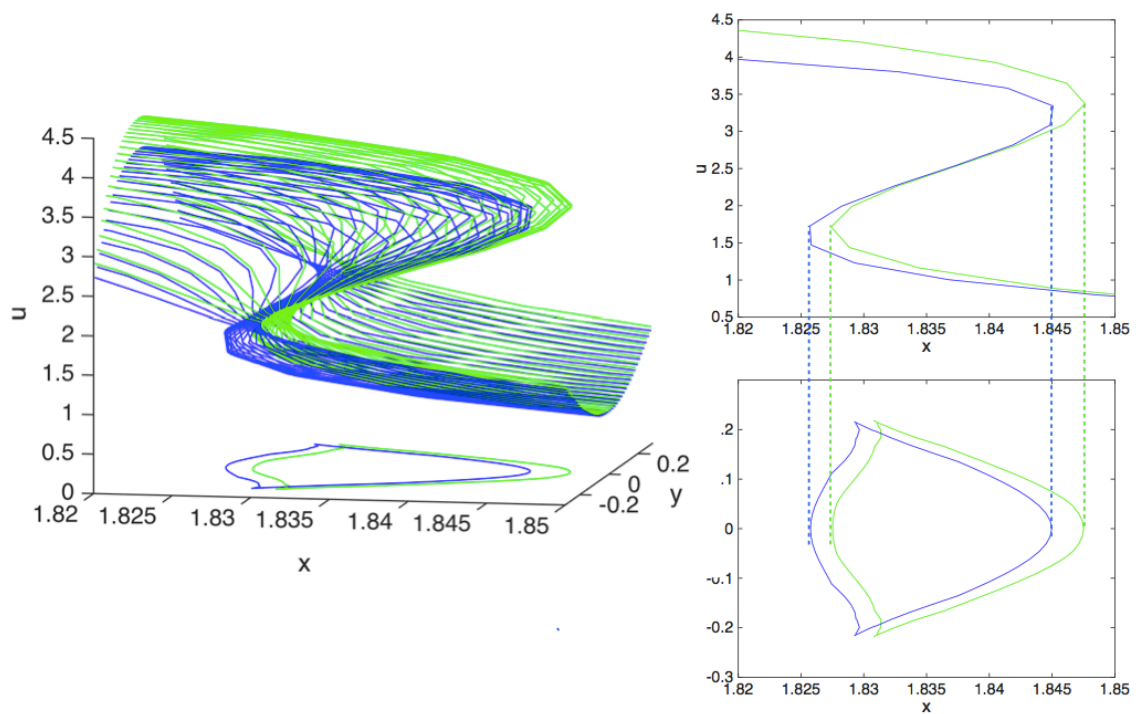

Figure 8. On the left, the solution $u(x, y, t)$ (blue line) of the dKP equation and its approximation (green line) (3.18) for $t=0.24>t_{c} \approx 0.222$. The regions of multivaluedness of the solutions are projected on the $(x, y)$-plane. On the right top: a cut through $u(x, y, t)$ along $y=0$. On the right bottom: the corresponding multivalued regions of $u(x, y)$ in the $(x, y)$-plane (blue line: numerical solution; green line: local approximation.)

We can now test to which extent the asymptotic description of the overturned region in Section 3, which only becomes exact in the limit $t \sim t_{c}$, can approximate our numerical results. Recall that the profile is described by (3.18), while the shape of the overturned region is given by $(3.23),(3.24)$. In Fig. 8 we show a comparison between a numerical solution of the dKP equation, obtained through the transformation (1.8) (blue), with the local approximate solution (3.18) shown in green. At $t=0.24$, i.e. shortly after overturning at $t_{c}=0.222$, there is good agreement in the description of the multivalued region. On the left, $u(x, y t)$ is shown in a perspective plot, on the top right an s-curve is produced by a cut along the $y=0$ plane. If corresponding cuts are considered for each value of $y$, a lip-shaped region is obtained inside which the profile has overturned (bottom right).

To test for the self-similar properties of the multivalued region, in Fig. 9 we show the numerical result as function of the rescaled coordinates $X_{1}, Y_{1}$, which are defined by (3.23) (red lines). Good agreement is seen with the asymptotic prediction (3.24) (blue lines), in particular for small values of three time distance $\bar{t}$ from the gradient catastrophe, as expected. The fact that the numerical results stay time independent to a good approximation demonstrates that the typical scales of the solution agree with the prediction $(3.23)$ : the width of the region scales like $\bar{t}^{3 / 2}$, its height like $\bar{t}^{1 / 2}$. 

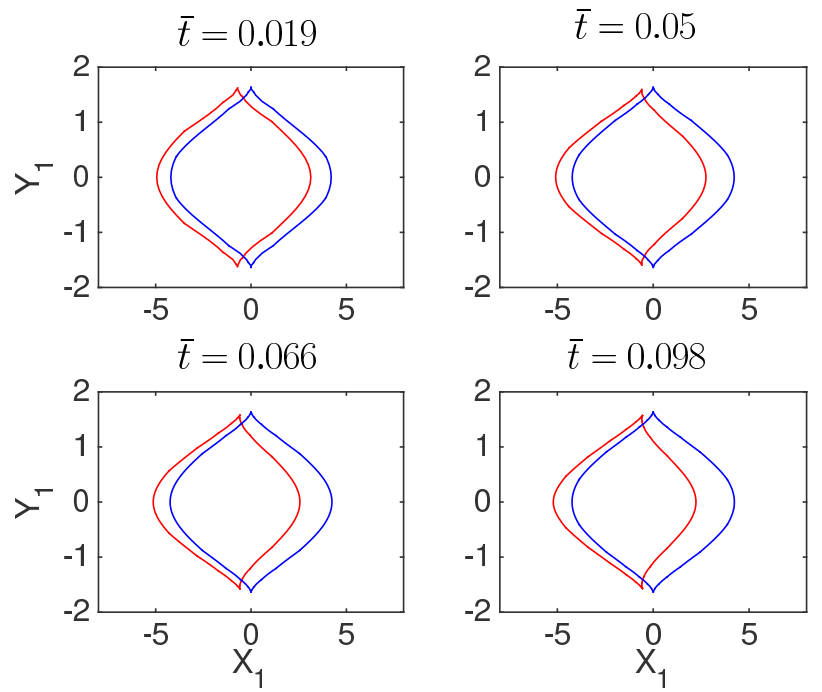

Figure 9. Multivalued region of the solution of the dKP equation as found from $\Delta(\xi, y, t)=0$ for the initial data (5.5). Results are written in selfsimilar rescaled coordinates $X_{1}$ and $Y_{1}$ defined by (3.23) for several values of $\bar{t}$ (red lines). The corresponding asymptotic boundary (3.24), shown in blue, is time-independent by construction.
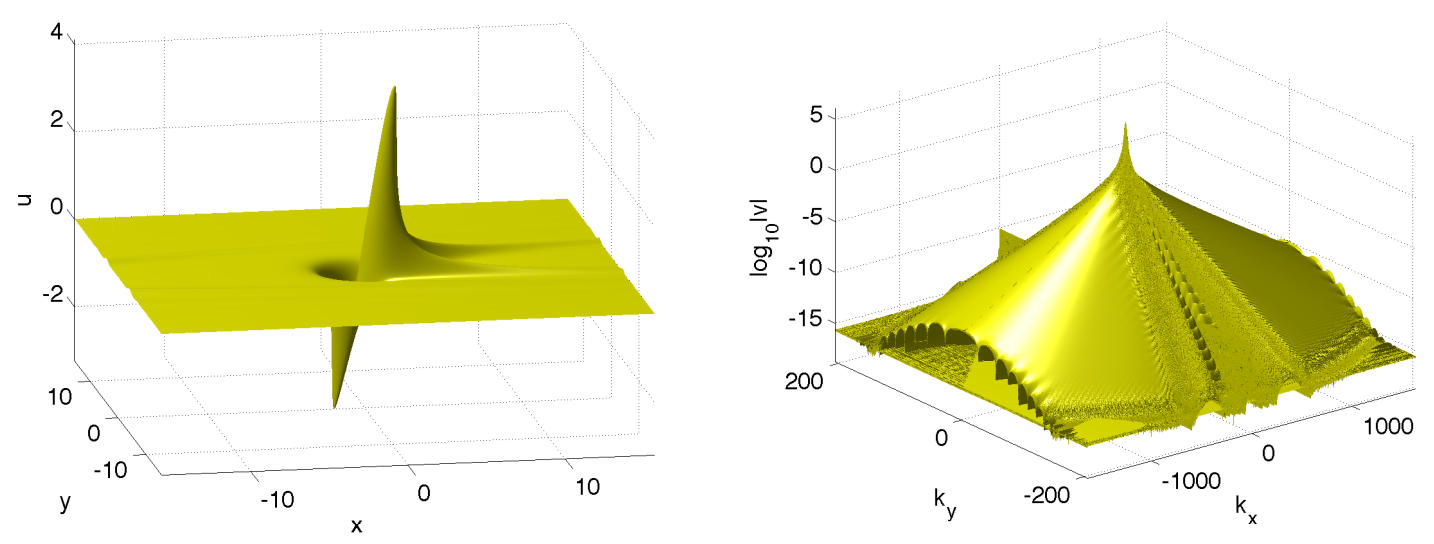

Figure 10. Numerical solution to the dissipative dKP equation $(5.2)$ with $c=0$ and $\epsilon=0.01$ for initial data (5.5) at time $t=0.32$ on the left, and the corresponding Fourier coefficients on the right.

We now turn to the numerical solution of the dissipative dKP equation (4.1), and to the comparison with our asymptotic theory, which is given by (4.27) in the general case, and by (4.28) for symmetric initial data. To resolve the strong gradients in the solutions to the dissipative dKP equation (5.2) that occur for small $\epsilon$, much higher resolution is needed than for the solution of (5.1) for the same initial data. For $\epsilon=0.01$ (with $c=0$ ) we use $N_{x}=2^{14}, N_{y}=2^{10}$ and $N_{t}=5000$ to find the solution of (5.2) with initial data (5.5) at $t=0.32$, shown in Fig. 10 on the left. At this value of $\epsilon$, the 

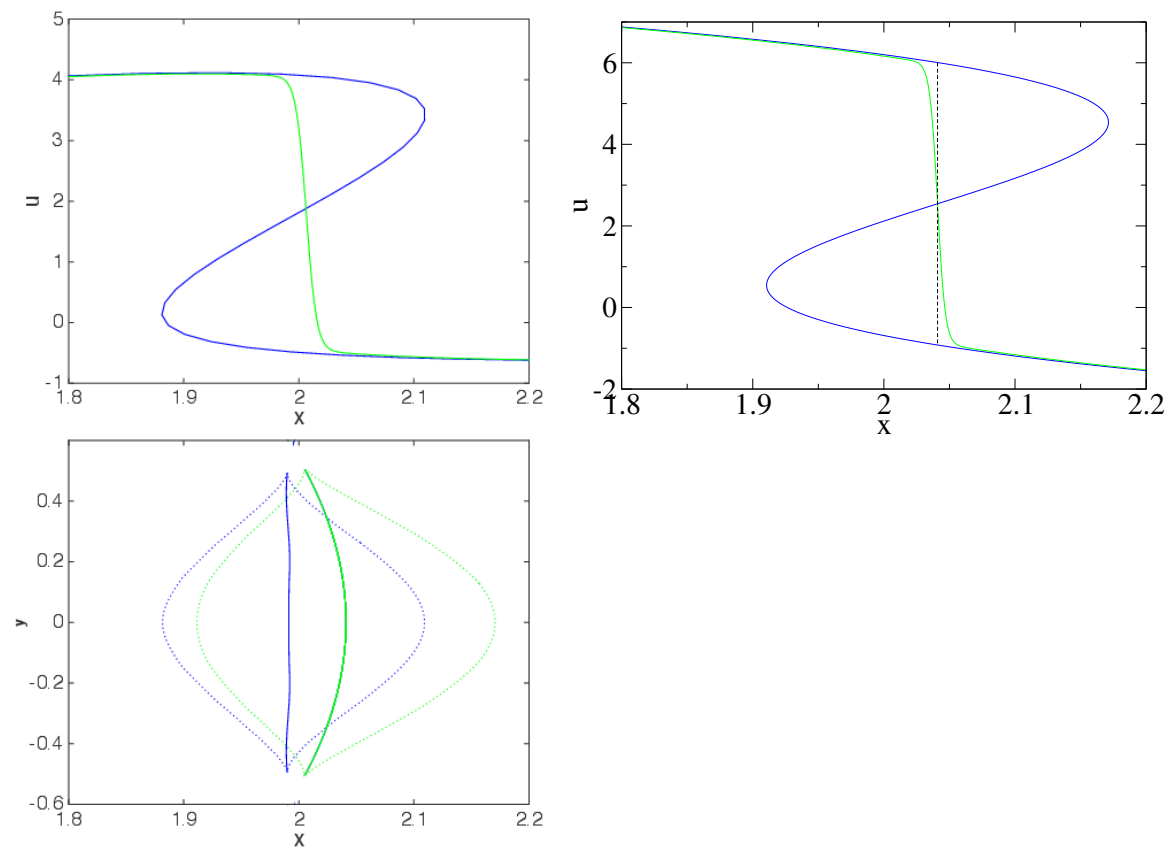

Figure 11. Top left: numerical solutions to the dKP equation (blue) and to the dissipative dKP equation (5.2) (green), for $c=0$ and $\epsilon=0.01$, using symmetric initial data (5.5). Shown is a slice along the line $y=0$ at $t=0.32>t_{c}=0.222$. Top right: the asymptotic approximations (3.16) and (4.27) to the same solutions; the dashed line marks the shock position $X=0$. Bottom: the dotted lines mark the multivalued regions for $t=0.32$, according to the numerical solution to the dKP equation (blue), and according to the asymptotic theory (3.26) (green). The green solid line is the asymptotic prediction for the shock front, as given by (4.6), and the blue solid line is a numerical estimate based on the inflection point of the dKP solution.

total loss of the $L^{2}$ norm (cf. 4.2 ) ) is of the order of $2 \%$. A comparison between the dKP solution and the Fourier coefficients, shown on the right, decay to below $10^{-10}$, as for the solutions to (5.1). To achieve higher resolutions, parallel computation would be needed.

In Fig. 11 (top left), we show a slice through the same dissipative solution at $y=0$ (green line), together with the corresponding dKP solution, which has become multivalued, as $\bar{t} \approx 0.1$. The dissipative solution exhibits a sharp front close to where the shock discontinuity is expected to be. Both curves are to be compared to our asymptotic results, shown on the top right, with the s-curve 3.18 shown in blue, and the dissipative asymptotics 4.28 in green. The sharp front is seen to be localized around the theoretical shock position, shown as the vertical dashed line. Since $\bar{t}$ is only moderately small, there exists a $30 \%$ difference in the height of the s-curve, 

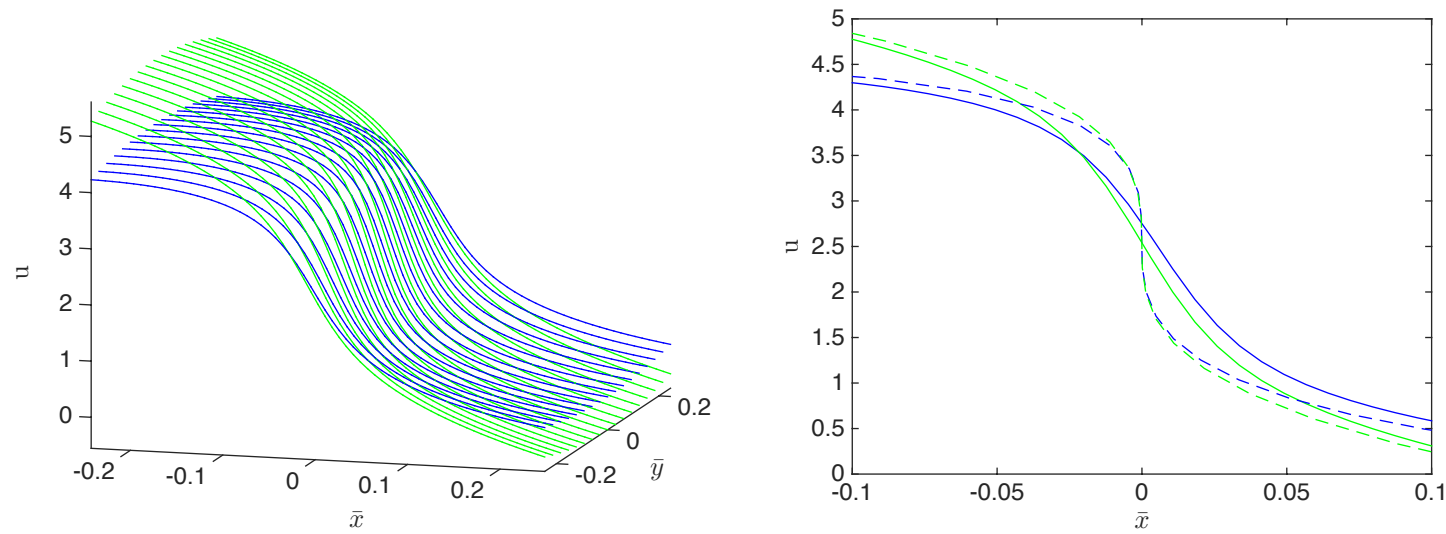

Figure 12. On the left, in blue the solution to the dissipative dKP equation 4.1 for $\epsilon=0.01$ and the symmetric initial data 5.5 at the critical time $t_{c}=0.222$ and near the critical point, and in green the asymptotic solution (4.27) given by the Pearcey integral. On the right the same plot along the line $y=0$. The dashed blue line is the solution of dKP equation and the green dashed line is the solution of the approximation (3.16) to the dKP solution.

but otherwise the overturning of the $\mathrm{dKP}$ equation is well reproduced. Within these limitations, the shape and width of the shock front, as well as the front position within the s-curve, are very well reproduced.

In the bottom graph of Fig. 11, we report the multivalued regions, as well as the position of the shock front, as given by the numerical solution (blue curves, with the shock front as the solid line), and our asymptotic theory (green curves, shock front solid). Once more, there is fair agreement in the shape and size of the lip-shaped multivalued regions (dashed lines), described by the dKP equation. The numerical shock position is estimated from the inflection point of the dKP solution, the theoretical prediction is the curve $X=0$.

In Fig. 12, we show the solution to the dissipative dKP equation (4.1) for $\epsilon=0.01$ and the asymptotic description $(4.27)$ for the symmetric initial data $(5.5)$ at the critical time in the vicinity of the critical point. While the asymptotic formula provides the best local approximation being best near the critical point, it can be seen to also correctly reproduce the $y$-dependence.

The approximation is also valid for small, nonzero values of $\bar{t}$ as can be seen in Fig. 13 where the same situation as in Fig. 12 is shown on the slice $y=0$ for several values of $\bar{t}$. 

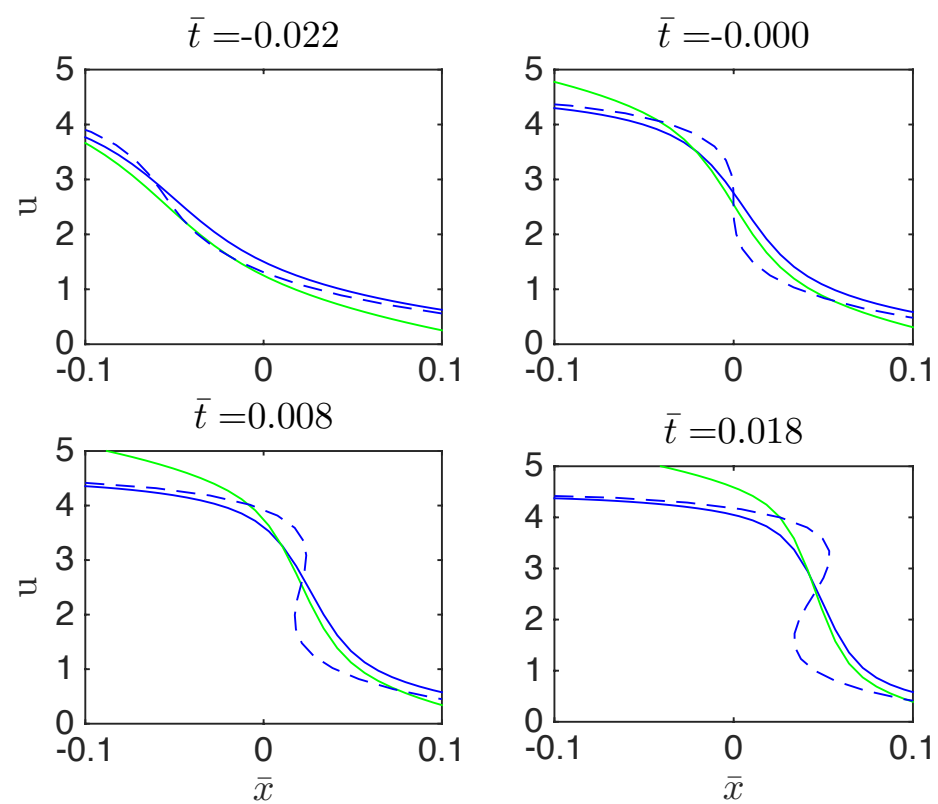

Figure 13. Solution to the dissipative dKP equation 4.1 for $\epsilon=0.01$ and the symmetric initial data 5.5 in blue, the Pearcey asymptotic solution (4.27) in green and the (weak) dKP solution dashed on the line $y=0$ for several values of $\bar{t}$.

\subsection{Nonsymmetric initial data}

In this section we consider two different initial profiles which are not symmetric with respect to $y \rightarrow-y$. The first,

$$
u(x, y, 0)=6 \partial_{x}\left\{(x+1)(y-1) e^{-x^{2}-y^{2}}\right\}
$$

still retains a radial symmetry for $x^{2}+y^{2} \rightarrow \infty$. As seen in Table 5.2, we can follow the evolution through two successive gradient catastrophes. The second profile,

$$
u(x, y, 0)=6 \partial_{x} e^{-x^{2}-5 y^{2}-3 x y}
$$

does not possess radial symmetry for large $x^{2}+y^{2}$, and we are able to compute the first gradient catastrophe only, whose critical parameters are also given in Table 5.2 .

To solve the Cauchy problem with initial data (5.6) for the dKP equation (2.8), we use $N_{x}=2^{9}$ and $N_{y}=2^{11}$ Fourier modes for $(x, y) \in[-5 \pi, 5 \pi]^{2}$ and $N_{t}=5000$ time steps for $t \leq 0.15$. The first critical time is reached at $t_{c}=0.08323 \ldots$, the second critical time is $\tilde{t}_{c}=0.1070 \ldots$; all other critical parameters are reported in Table 5.2 . The relative computed $L^{2}$ norm is conserved to the order of $10^{-14}$, and the Fourier coefficients decrease to the order of the Krasny filter as can be seen in Fig. 14 (left). 


\begin{tabular}{|c|c|c|c|c|c|c|}
\hline Breaking events & Initial data & $t_{c}$ & $x_{c}$ & $y_{c}$ & $u_{c}$ & $\xi_{c}$ \\
\hline First breaking & $6 \partial_{x}\left\{(x+1)(y-1) e^{-x^{2}-y^{2}}\right\}$ & 0.0832 & -1.210 & -0.368 & -4.958 & -0.798 \\
\hline Second breaking & $6 \partial_{x}\left\{(x+1)(y-1) e^{-x^{2}-y^{2}}\right\}$ & 0.1070 & 2.004 & -0.368 & 4.4066 & 1.534 \\
\hline First Breaking & $6 \partial_{x}\left(e^{-x^{2}-5 y^{2}-3 x y}\right)$ & 0.086 & 0.088 & -0.245 & -1.477 & 0.215 \\
\hline
\end{tabular}

Table 2. Critical parameters for the first two wave breaking events, with weakly asymmetric initial data (5.6). For the strongly asymmetric initial data (5.7) only the first breaking could be computed.
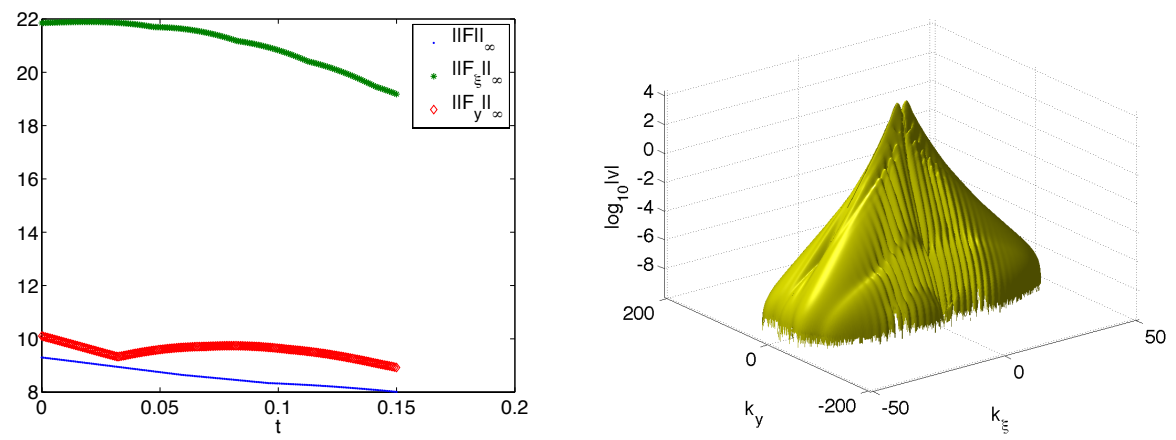

Figure 14. Same as Fig. 6, but with initial data (5.6) (left). The Fourier coefficients on the right are shown for $t=0.15$.

As seen in the same figure on the left, the $L^{\infty}$ norm of the solution $F$ and the norm of its gradient also appear to decrease for large $t$, so again there is no indication of a blow-up of the solution. However, to be able to run the code for longer times, larger computational domains would have to be used.

On the left of Fig. 15, we trace the boundary of the multivalued regions of $u(x, y, t)$ at four times shortly after the first gradient catastrophe; the times $\bar{t}$ relative to the singularity are reported on the top of each graph. On the right of the same figure, the same multivalued regions are plotted as functions of the rescaled coordinates $X_{1}$ and $Y_{1}$ defined in 3.23). Once more, in the rescaled coordinates the shape of the multivalued region is almost constant, and agrees well with the theoretical prediction, shown in blue. Note the slight asymmetry of the lip shape with respect to the reflection symmetry $y \rightarrow-y$.

For the initial data (5.7), the code is run with $N_{x}=N_{y}=2^{11}$ Fourier modes on 

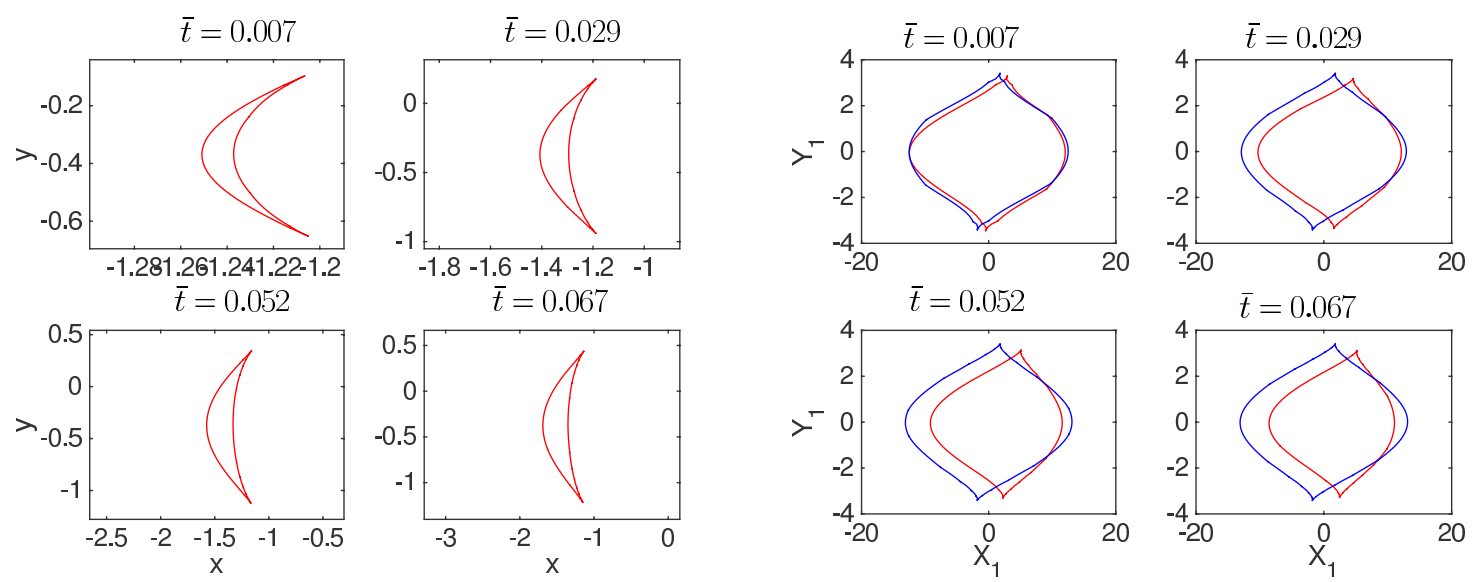

Figure 15. Left: boundary of the multivalued region found from a numerical solution to the dKP equation for the initial data (5.6), for several values of $t>t_{c}=0.08323 \ldots$ in the original $(\mathrm{x}, \mathrm{y})$ variables. Right: The red boundaries on the right are the same data represented in self-similar variables $X_{1}$ and $Y_{1}$ as defined in (3.23), predicted to be time-independent by our asymptotic theory. The corresponding self-similar boundary, given by (3.24), is plotted in blue.
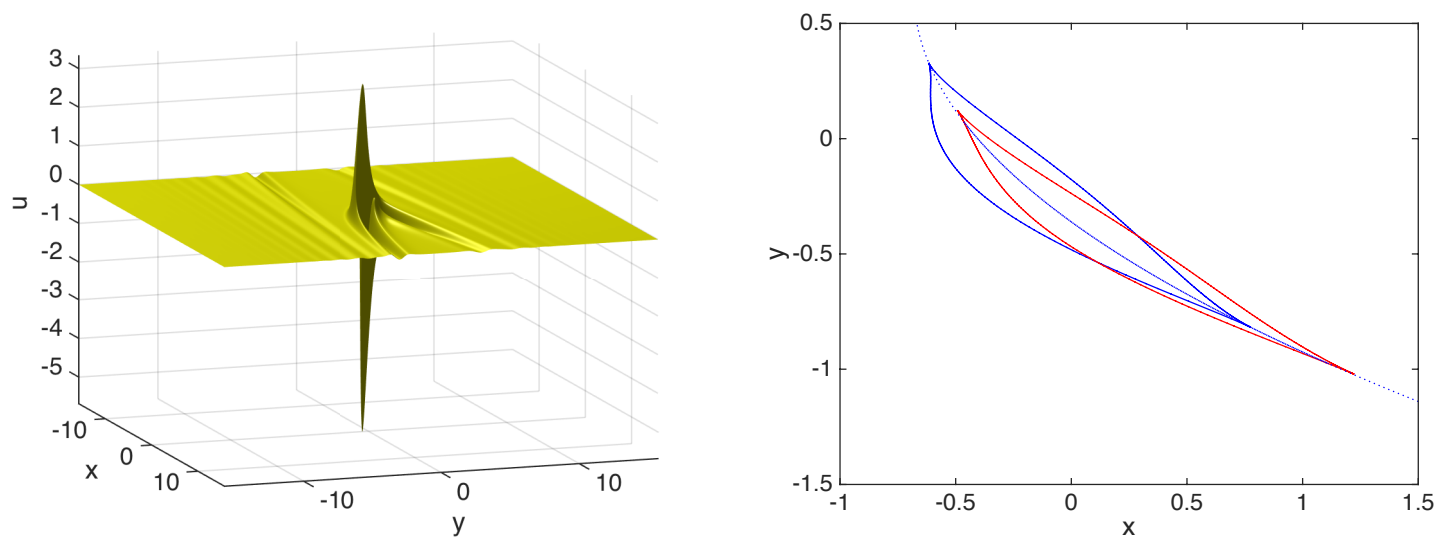

Figure 16. Left: numerical solution to the dKP equation 2.1) for strongly asymmetric initial data (5.7) at $t=0.15>t_{c}=0.087$. Right: The corresponding contour of the multivalued region $\Delta(\xi, y, t)=0$ (red), compared to the asymptotic theory (3.24) (blue); the dashed line corresponds to $X=0$ as given by 4.6).

the same spatial domain as before, using $N_{t}=2000$ time steps for $t \leq 0.15$. The first gradient catastrophe is found at $t_{c}=0.087 \ldots$, see Table 5.2 for the remaining critical parameters. The solution at the final time (cf. Fig. 16, left) is strongly asymmetric. This also implies an asymmetry of the tails of the solution and thus a stronger effect of the algebraic decay of the solution towards spatial infinity. The asymmetry of the tails of the solution also affects the Fourier coefficients. Despite a higher resolution than that of Fig. 14, there are small contributions to the high wave number Fourier coefficients 

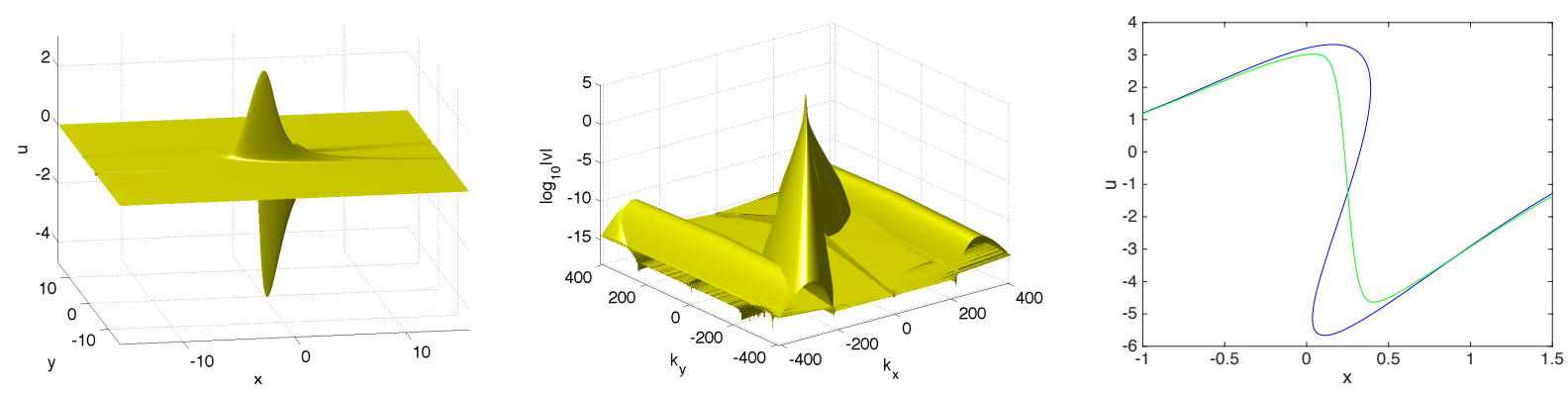

Figure 17. Left: numerical solution to the dissipative dKP equation $(5.2)$ with $\epsilon=0.04, c=1$, for initial data (5.7), at $t=0.15$. Center: the corresponding Fourier coefficients. Right: a slice of the left plot along the line $y=-0.4985$ (green), together with the corresponding solution of the dKP equation (blue).

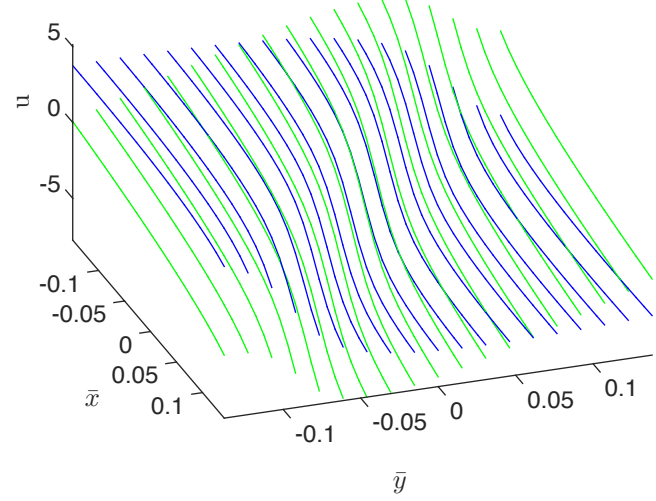

Figure 18. In blue the solution of the dissipative dKP equation and in green the Pearcey asymptotic solution 4.27) for $\epsilon=0.01$ and the strongly asymmetric initial data (5.7) at the critical time $t_{c}$ and near the critical point of the dKP solution.

along the $k_{y}$ axis above the Krasny filter, which eventually cause the numerical scheme to break down. As a result, we do not reach a second catastrophe in this example. At $t=0.15$, the relative computed $L^{2}$ norm is still conserved with an accuracy in the order of $10^{-13}$. The $L^{\infty}$ norm of $F$ and of its gradient do not indicate blow-up, but they are also not decreasing. If the solution exists for large $t$ also, then the computation did not reach the asymptotic regime.

The asymmetry of the solution can also clearly be seen from the contour delimiting the multivalued region, seen as the red line in Fig. 16 (right). This is compared to the asymptotic theory at $\bar{t}=0.063$, shown as the blue line. Theory correctly describes the strong asymmetry and the orientation of the lip shape, but there are some quantitative differences. This indicates that the size of the critical region is smaller in the case of strong asymmetry. 
For the dissipative dKP equation for the initial data (5.7), we consider $\epsilon=0.04$ to obtain the solution shown in Fig. 17 on the left. The Fourier coefficients in the middle of the same figure are also rather asymmetric, but decrease to the order of the Krasny filter. Due to the higher value of $\epsilon$, the loss of the $L^{2}$ norm is of the order of $22.2 \%$. On the right of Fig. 17, we compare the dissipative solution to the corresponding solution of the dKP equation. Although the width of the front is greater, owing to a higher value of $\epsilon$, it is set inside the s-curve where the shock position is expected to be.

In Fig. 18 we show the dissipative dKP equation 4.1 for $\epsilon=0.01$ for initial data (5.7). While in the symmetric case $F_{y}^{c}=0$, here we have $F_{y}^{c} \approx-17.39$, consistent with a strongly asymmetric shock. Even in this case, the full two-dimensional structure of the step is well described by the asymptotic theory.

\section{Conclusions}

We have introduced a coordinate transformation, inspired by the method of characteristics, to investigate wave breaking in the dispersionless KadomtsevPetviashvili equation. As a result, the entire region where the profile is overturned is mapped onto a smooth and single valued function. The transformed equation remains smooth near the gradient catastrophe. Moreover, our numerics show that solutions remain smooth even beyond secondary wave breaking events. This permits us to compute solutions up to the first gradient catastrophe with much reduced numerical effort, and then to continue into the overturned region, where direct numerical simulations of the dKP equation fail. From the overturned profile, one can reconstruct the shock position, using the jump condition (4.5).

Using the fact that the transformed profile remains smooth at the gradient catastrophe, we have calculated the local similarity form of the profile. This allows us to calculate the lip shape of the overturned region analytically, and to find the position of shock. Both the shape and the scaling properties of this region agree well with numerical simulations.

We have also investigated the dissipative version of the dKP equation, which regularizes the gradient catastrophe. We performed direct numerical simulations of this equation for small dissipation, which we continued beyond the first gradient catastrophe. Results agree with expected shock solutions, except that the jump at the shock position is replaced by a smooth but rapidly varying profile. To investigate the shape of this profile, we use our characteristic transformation to map the dissipative KP equation locally to Burgers' equation, which we can solve to obtain a local similarity description of the profile in two dimensions. Asymptotic analysis leads to a description of the profile in terms of Pearcey's function, which is in good agreement with numerics. 
We believe that the methods developed in this paper are of interest to study shock formation in a wider class of hyperbolic equations, including the compressible Euler equation. Here a significant complication lies in the fact that there are two families of characteristics in the corresponding one-dimensional problem, and hence a transformation based on a single characteristic cannot be expected to lead to a solution which avoids overturning for all times. However, shocks are generically expected to form with respect to one of the two characteristics only [30], so a transformation such as (1.8) will still be able to unfold the profile locally. However, the necessary transformation will depend on which of the characteristics is involved, and thus implicitly on initial conditions.

\section{Acknowledgments}

JE's work was supported by a Leverhulme Trust Research Project Grant. TG was partially supported by Miur Research project Geometric and analytic theory of Hamiltonian systems in finite and infinite dimensions of Italian Ministry of Universities and Research.

[1] S. Alinhac, Blowup for nonlinear hyperbolic equations, Birkhäuser, 1995.

[2] _ Hyperbolic partial differential equations, Springer, Dordrecht, 2009.

[3] V. I. Arnold, Catastrophe theory, Springer, 1984.

[4] _ Singularities of caustics and wave fronts, Kluwer, 1990.

[5] A. Arsie, P. Lorenzoni, and A. Moro, Integrable viscous conservation laws. Nonlinearity 28 (2015), no. $6,1859-1895$.

[6] S. Bianchini and A. Bressan, Vanishing viscosity solutions of nonlinear hyperbolic systems, Ann. of Math. 161 (2005), 223-342.

[7] M. Birem and C. Klein, Multidomain spectral method for Schrödinger equations, (2014).

[8] E. Brézin, E. Marinari, and G. Parisi, A non-perturbative ambiguity free solution of a string model, Phys. Lett. B 242 (1990), 35.

[9] A. Chorin and J. E. Marsden, A mathematical introduction to fluid mechanics, Springer, 2000.

[10] S. Cox and P. Matthews, Exponential time differencing for stiff systems, J. Comp. Phys. 176 (2002), 430-455.

[11] NIST Digital Library of Mathematical Functions, http://dlmf.nist.gov/, Release 1.0.9 of 2014-0829, Online companion to 42 .

[12] B. Dubrovin and M. Elaeva, On the critical behavior in nonlinear evolutionary PDEs with small viscosity, Russ. J. Math. Phys. 19 (2012), 449-460.

[13] M. Dunajski, L. Mason, and P. Tod, Einstein-Weyl geometry, the dKP equation and twistor theory., J. Geom. Phys. 37 (2001), 63-93.

[14] J. Eggers and M. A. Fontelos, The role of self-similarity in singularities of partial differential equations, Nonlinearity 22 (2009), R1.

[15] _ Cusps in interfacial problems, Panoramas et Synthèses 38 (2013), 69.

[16] E.V. Ferapontov, A. Moro, and V.V. Sokolov, Hamiltonian systems of hydrodynamic type in $2+1$ dimensions., Comm. Math. Phys. 285 (2009), 31-65.

[17] J. Gibbons and Y. Kodama, A method for solving the dispersionless KP hierarchy and its exact solutions, Phys. Lett. A 135 (1989), 167-180. 
[18] J. Goodman and Z. Xin, Viscous limits for piecewise smooth solutions to systems of conservation laws, Arch. Rational Mech. Anal. 121 (1992), 235-265.

[19] O. Gués, G. Métivier, M. Williams, and K. Zumbrun, Nonclassical multidimensional viscous and inviscid shocks, Duke Math. J. 142 (2008), 1-110.

[20] E. Hopf, The partial differential equation $u_{t}+u u_{x}=\mu u_{x x}$, Comm. Pure Appl. Math. 3 (1950), 201-230.

[21] A. M. Il'in, Matching of asymptotic expansions of solutions of boundary value problems, vol. 102, AMS, 1992.

[22] B. B. Kadomtsev and V. I. Petviashvili, On the stability of solitary waves in weakly dispersive media, Sov. Phys. Dokl. 15 (1970), 539.

[23] C. Klein, Fourth order time-stepping for low dispersion Korteweg-de Vries and nonlinear Schrödinger equation, ETNA 29 (2008), 116-135.

[24] C. Klein and K. Roidot, Fourth order time-stepping for kadomtsev-petviashvili and daveystewartson equations, SIAM J. Sci. Comput. 33 (2011), 3333-3356.

[25] _ Numerical study of shock formation in the dispersionless kadomtsev-petviashvili equation and dispersive regularizations, Physica D 265 (2013), 1-25.

[26] C. Klein, C. Sparber, and P. Markowich, Numerical study of oscillatory regimes in the kadomtsevpetviashvili equation, J. Nonl. Sci. 17 (2007), 429-470.

[27] B. Konopelchenko, L. Martínez Alonso, and O. Ragnisco, The $\bar{\partial}$-approach to the dispersionless KP hierarchy, J. Phys. A 3447 (2001), 10209-10217.

[28] R. Krasny, A study of singularity formation in a vortex sheet by the point-vortex approximation, J. Fluid Mech. 167 (1986), 65-93.

[29] S. Kruzhkov, First-order quasilinear equations with several space variables, Math. USSR Sbornik 10 (1970), 217-243.

[30] L. D. Landau and E. M. Lifshitz, Fluid mechanics, Pergamon: Oxford, 1984.

[31] P.D. Lax, Hyperbolic systems of conservation laws ii, Comm. Pure Appl. Math. 10 (1957), 537566.

[32] R. J. LeVeque, Numerical methods for conservation laws, Springer, 1992.

[33] A. Majda, Compressible fluid flow and systems of conservation laws in several space variables, Springer-Verlag, New York, 1984.

[34] S. V. Manakov and P. M. Santini, The Cauchy problem on the plane for the dispersionless Kadomtsev-Petviashvili equation, JETP Letters, 83, (2006), No 10, 462-466.

[35] _ On the solutions of the dKP equation: the nonlinear riemann hilbert problem, longtime behaviour, implicit solutions and wave breaking,J. Phys. A: Math. Theor. 41 (2008) 055204, $23 \mathrm{pg}$.

[36] _ solutions, the Cauchy problem for small initial data and wave breaking, J. Phys. A: Math. Theor. 44 (2011), 405203.

[37] _ Solvable vector nonlinear Riemann problems, exact implicit solutions of dispersionless PDEs and wave breaking, J. Phys. A: Math. Theor. 44, (2011) 345203 (19pp), doi:10.1088/17518113/44/34/345203. arXiv:1011.2619.

[38] _ Wave breaking in the solutions of the dispersionless Kadomtsev-Petviashvili equation at a finite time, Theoret. and Math. Phys. 172 (2012), 1117.

[39] W. L. Marar and J. J. Nuño-Ballesteros, Slicing corank 1 map germs from $\mathbb{C}^{2}$ to $\mathbb{C}^{3}$, Q. J. Math. 650 (2014), 1375.

[40] L. Molinet, J.-C. Saut, and N. Tzvetkov, Well-posedness and ill-posedness results for the Kadomtsev-Petviashvili-I equation, Duke Math. J. 115 (2002), 353-384.

[41] J. Nye, Natural focusing and fine structure of light: Caustics and wave dislocations, Institute of Physics Publishing, Bristol, 1999. 
[42] F. W. J. Olver, D. W. Lozier, R. F. Boisvert, and C. W. Clark (eds.), NIST Handbook of Mathematical Functions, Cambridge University Press, New York, NY, 2010, Print companion to [11.

[43] Y. Pomeau, M. Le Berre, P. Guyenne, and S. Grilli, Wave-breaking and generic singularities of nonlinear hyperbolic equations, Nonlinearity 21 (2008), T61-T79.

[44] T. Poston and I. Stewart, Catastrophe theory and its applications, Dover Publications, Mineola, 1978.

[45] A.Raimondo, Frobenius manifold for the dispersionless Kadomtsev-Petviashvili equation. Comm. Math. Phys. 311 (2012), no. 3, 557594.

[46] A.Rozanova, The Khokhlov-Zabolotskaya-Kuznetsov equation, C. R. Math. Acad. Sci. Paris 344 (2007), no. 5, 337 - 342 .

[47] K. Takasaki and T. Takebe, Integrable hierarchies and dispersionless limit., Rev. Math. Phys. 5 (1995), 743-808.

[48] G. B. Whitham, Linear and nonlinear waves, John Wiley \& Sons, 1974.

[49] E. A. Zabolotskaya and R. V. Khokhlov, Quasi-plane waves in the nonlinear acoustics of confined beams, Sov. Phys. Acoustics 15 (1969), 35-40.

[50] V.E. Zakharov and S.V. Manakov, Construction of multidimensional nonlinear integrable systems and their solutions, Funktsional. Anal. i Prilozhen 19 (1985), 11-25. 